\title{
Applications of the Atmospheric Transport and Diffusion of LES Modeling to the Spread and Dissipation of COVID-19 Aerosol Particles inside and outside the Japan National Stadium (Tokyo Olympic Stadium)
}

\author{
Takanori Uchida $\mathbb{D}^{1}$ and Ryo Araya $\mathbb{D}^{2}$ \\ ${ }^{1}$ Research Institute for Applied Mechanics (RIAM), Kyushu University, 6-1 Kasuga-kouen, Kasuga, Fukuoka 816-8580, Japan \\ ${ }^{2}$ Environmental GIS Laboratory Co. Ltd., Japan \\ Correspondence should be addressed to Takanori Uchida; takanori@riam.kyushu-u.ac.jp
}

Received 8 September 2020; Revised 9 March 2021; Accepted 9 April 2021; Published 9 June 2021

Academic Editor: Luis Carlos Rabelo

Copyright (c) 2021 Takanori Uchida and Ryo Araya. This is an open access article distributed under the Creative Commons Attribution License, which permits unrestricted use, distribution, and reproduction in any medium, provided the original work is properly cited.

\begin{abstract}
In this paper, we use an analysis function for gas diffusion known as the Research Institute for Applied Mechanics, Kyushu University, Computational Prediction of Airflow over Complex Terrain (RIAM-COMPACT), which was developed for complex terrain, in Airflow Analyst software, and apply it to the spread and dissipation of a fluid layer (assuming the fluid layer contains COVID-19 particles). First, to verify the prediction accuracy of the gas diffusion using RIAM-COMPACT, comparisons with past wind tunnel test results conducted on simple and complex terrains are presented under neutral atmospheric stability. The results of the numerical simulations carried out in this study show good agreement with the wind tunnel experiments for both simple and complex terrains. Next, a model of the Japan National Stadium (Tokyo Olympic Stadium) was constructed using 3D detailed topographic Advanced World 3D Map (AW3D) data generated by combining high-resolution satellite images. We tried to reproduce the hypothetical spread and dissipation of the fluid layer (assuming the fluid layer contains COVID-19 particles) inside and outside of the Japan National Stadium using Airflow Analyst implemented with the RIAM-COMPACT analysis function for gas diffusion. We paid special attention to the effect of wind ventilation driven by natural wind. The numerical results under various scenarios show that ventilation driven by natural wind is very effective for the Japan National Stadium.
\end{abstract}

\section{Introduction}

Currently, computational fluid dynamics (CFD) techniques have entered the stage of practical application in various fields [1-9]. Over many years, our research group has developed a CFD technique [10-14] that can predict atmospheric flow and gas diffusion over a steep complex terrain under different thermal stratifications with high accuracy, focusing on microscale to mesoscale information. This CFD technique is called the Research Institute for Applied Mechanics, Kyushu University, Computational Prediction of Airflow over Complex Terrain (RIAM-COMPACT) [10-14] and adopts large-eddy simulation (LES) as a turbulence model. Many research results have already been reported using the LES approach based on RIAM-COMPACT, including (1) the mechanism of a local strong wind induced by a topographic effect [10]; (2) topographic speed-up effects [11]; (3) wind turbine failures due to terrain-induced turbulence [12]; (4) the aerodynamics of horizontal axis wind turbine wakes [13]; and (5) the transport and dispersion of air pollutants under neutral, stable, and unstable atmospheric conditions [14, 15].

In parallel with the above, we are also currently developing new software based on CFD techniques focusing on wind environment assessment in urban areas [16]. In particular, this CFD software has a deep affinity with the Geographic Information System (GIS) technique. Our CFD software is 


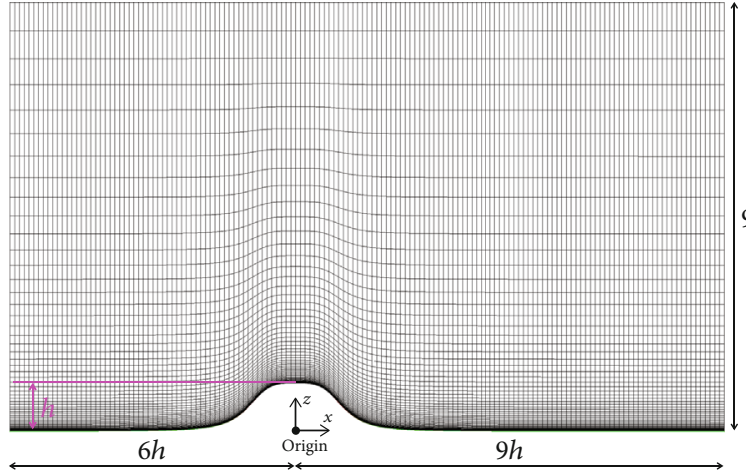

(a)

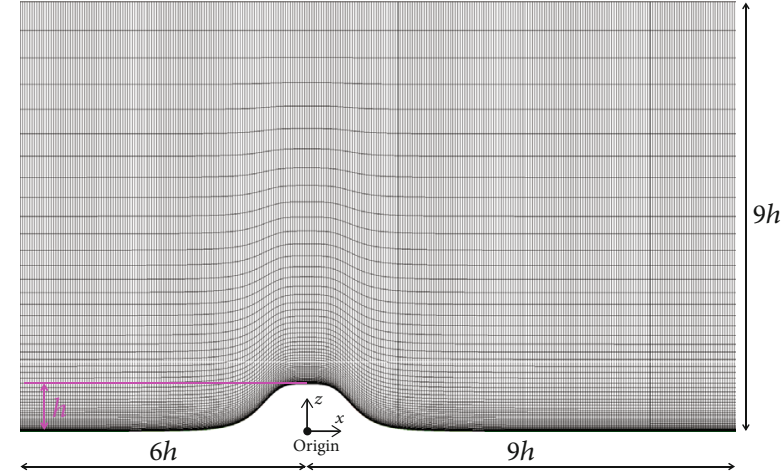

(b)

Figure 1: Computational grid in the $x-z$ plane: (a) Case 1 and Case 2; (b) Case 3.

TABLe 1: Parameters in the study simulations.

\begin{tabular}{|c|c|c|c|}
\hline & Case 1 & Case 2 & Case 3 \\
\hline Domain size & \multicolumn{3}{|c|}{$15 h(x) \times 10 h(y) \times 9 h(z)$, where $h$ is the hill height } \\
\hline Grid points & $151(x)$ & $\times 61(z)$ & $301(x) \times 201(y) \times 61(z)$ \\
\hline \multirow{2}{*}{ Grid width in the $x$ and $y$ directions (uniform width) } & \multicolumn{2}{|c|}{$\Delta x=0.1 h$} & $\Delta x=0.05 h$ \\
\hline & \multicolumn{2}{|c|}{$\Delta y=0.1 h$} & $\Delta y=0.05 h$ \\
\hline Grid width in the $z$ direction (nonuniform width) & \multicolumn{3}{|c|}{$\Delta z=3.5 \times 10^{-3}-6.0 \times 10^{-1} h$} \\
\hline Nondimensional time increment & \multicolumn{2}{|c|}{$\Delta t=2.0 \times 10^{-3}$} & $\Delta t=1.0 \times 10^{-3}$ \\
\hline Nondimensional time averaging & $t=100.0-200.0$ & $t=100.0-1000.0$ & $t=100.0-200.0$ \\
\hline $\begin{array}{l}\text { Reynolds number defined by the height } h \text { of the } \\
\text { isolated hill and the uniform flow } U\end{array}$ & \multicolumn{3}{|c|}{$1.0 \times 10^{4}$} \\
\hline
\end{tabular}

called Airflow Analyst and runs on ArcGIS, which is generalpurpose GIS software sold by Environmental Systems Research Institute, Inc. (ESRI) [17] in the United States. Airflow Analyst has many advantages, including the following:

(1) A significant reduction in the required time related to 3D city data construction by utilizing paid and free geospatial data resources that are generally distributed

(2) A significant reduction in the required time for $3 \mathrm{D}$ city data construction due to the rapid progress in data compatibility with computer-aided design (CAD), building information modeling (BIM), construction information modeling (CIM), and GIS

(3) It is possible to easily import 3D city data constructed based on satellite photos, aerial photos, and unmanned aerial vehicle (UAV) photos, as well as more sophisticated 3D city data created from lasermeasured point cloud data

(4) It is possible to set the wind direction to be calculated, as well as the computational domain and the conditions for grid generation, intuitively and in a short period of time when confirming the actual scale on the map

(5) The simulation results are visualized threedimensionally on a general map because this map holds spatial reference information, such as coordinate information. Furthermore, spatial analysis that overlaps with other spatial information is also possible

(6) It is simple to distribute or share a series of calculation results by overlaying them on a map service on the Web

In this paper, we apply the RIAM-COMPACT analysis function for gas diffusion, which was developed for complex terrain, to Airflow Analyst and use it to study the spread and dissipation of the fluid layer (assuming the fluid layer contains COVID-19 particles). First, to verify the prediction accuracy of the gas diffusion using RIAM-COMPACT, comparisons with past wind tunnel test results conducted on simple and complex terrain were conducted. Next, a model of the Japan National Stadium (Tokyo Olympic Stadium) was constructed using 3D detailed topographic Advanced World 3D Map (AW3D) data [18] generated by combining highresolution satellite images. We sought to reproduce the hypothetical spread and dissipation of the fluid layer (assuming the fluid layer contains COVID-19 particles [19]) inside and outside of the Japan National Stadium using Airflow Analyst implemented with the RIAM-COMPACT analysis function for gas diffusion. We paid special attention to the effect of ventilation driven by natural wind. 


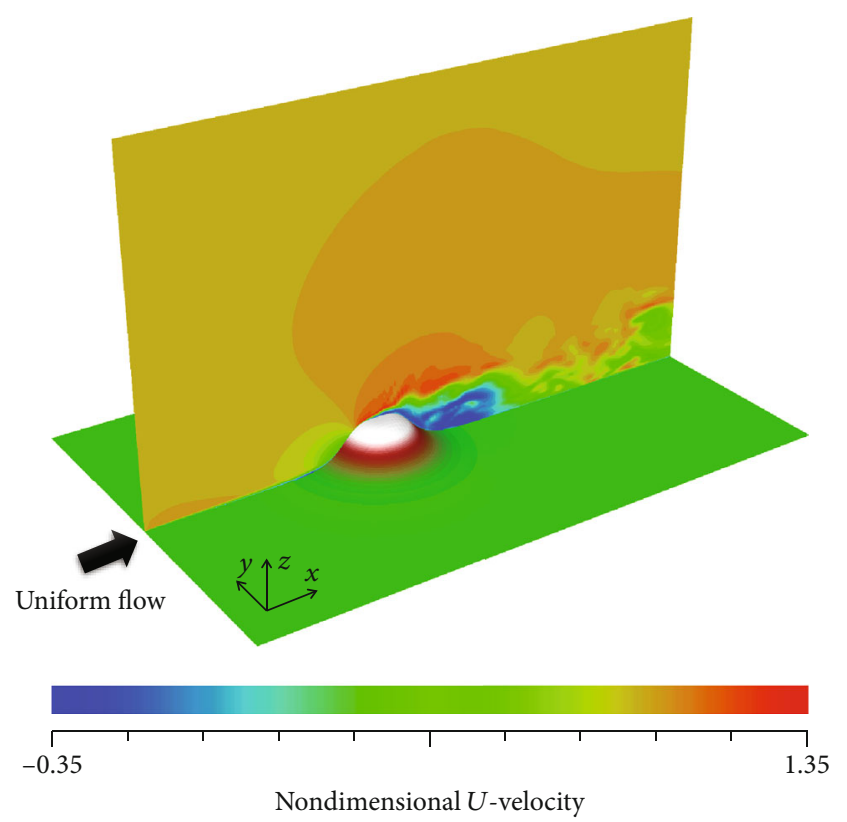

(a)
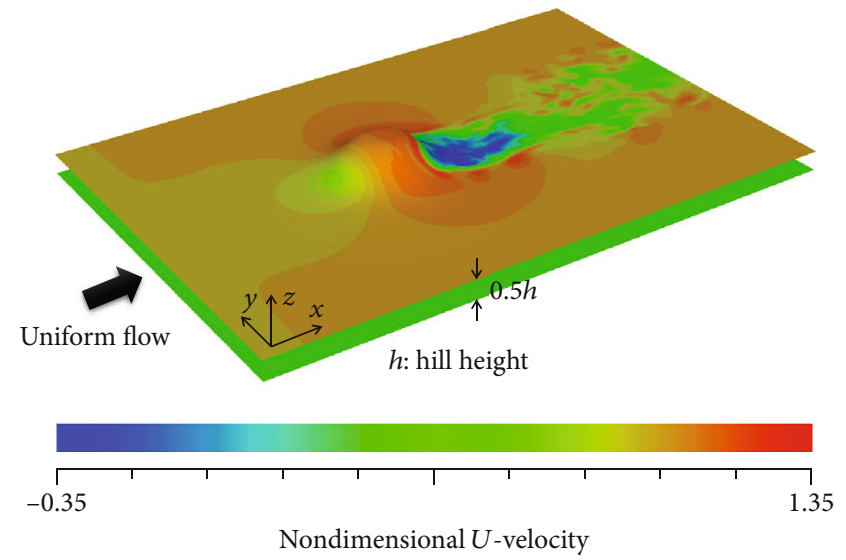

(b)

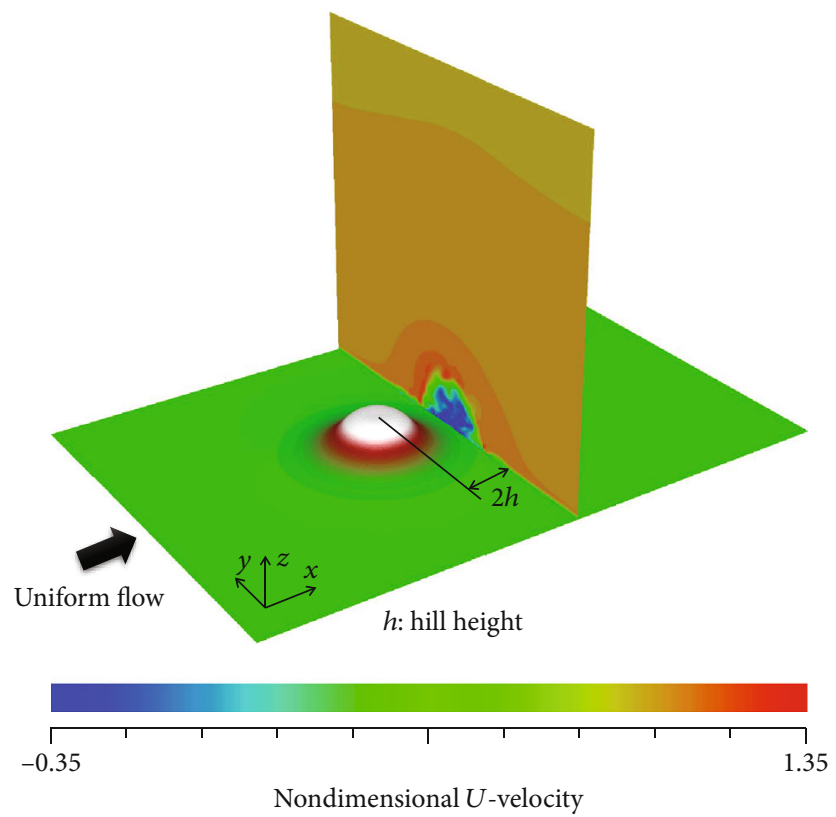

(c)

Figure 2: Spatial distribution of the instantaneous nondimensional $U$-velocity in Case 1 (nondimensional time $t=100$ ): (a) $x$ - $z$ plane; (b) $x-y$ plane; (c) $y-z$ plane.

\section{Summary of the In-House LES Solver}

For the numerical simulations, a collocated grid in a general curvilinear coordinate system and a staggered grid in a Cartesian orthogonal coordinate system were adopted. For the numerical technique, the finite-difference method (FDM) was adopted, and a large-eddy simulation (LES) model was used for the turbulence model. In the LES model, a spatial filter was applied to the flow field to separate eddies of various scales into grid-scale (GS) components that were larger than the computational grid cells and subgrid-scale (SGS) compo- nents, which were smaller than the computational grid cells. Large-scale eddies, i.e., the GS components of turbulence eddies, were directly numerically simulated without the use of a physically simplified model. In contrast, the dissipation of energy, which is the main effect of small-scale eddies (i.e., the SGS components), was modeled according to a physics-based analysis of SGS stress.

For the governing equations of flow in tensor form $(i, j=1,2,3)$, a filtered continuity equation for incompressible fluid (equation (1)) and a filtered Navier-Stokes equation (equation (2)) were used. When it became necessary to 


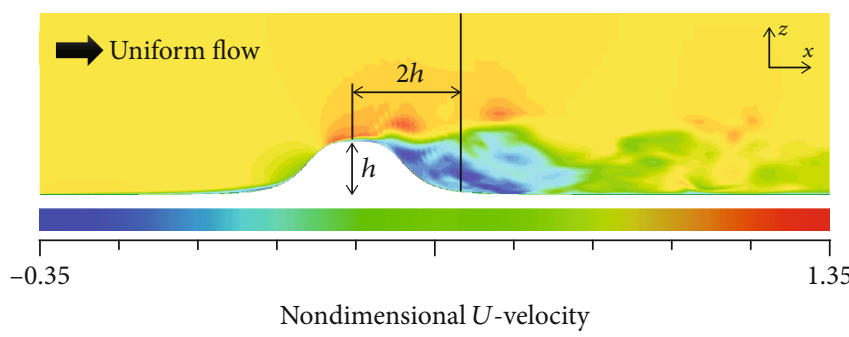

(a)

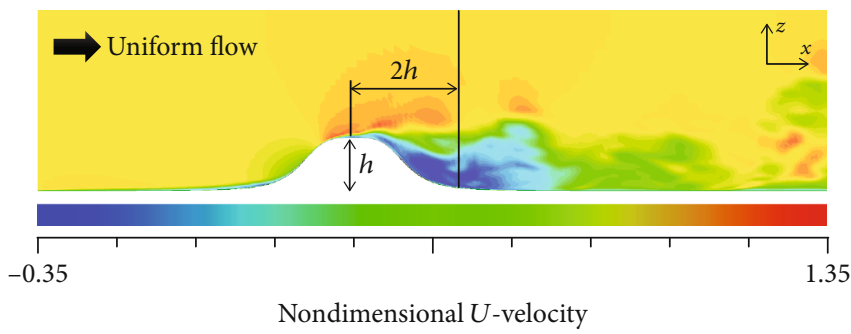

(c)

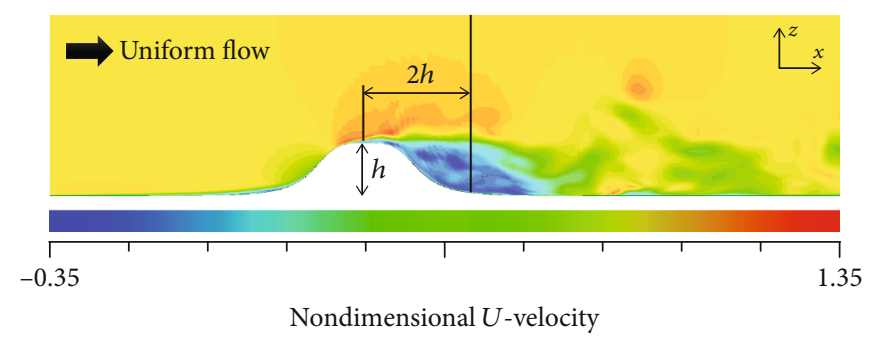

(b)

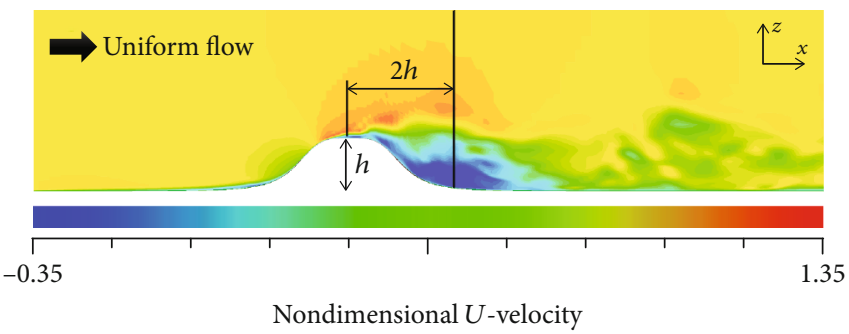

(d)

FIGURE 3: Spatial distribution of the instantaneous nondimensional $U$-velocity in the $x$ - $z$ plane under Case 1: (a) nondimensional time $t=107$ ; (b) $t=112$; (c) $t=117$; (d) $t=122$.

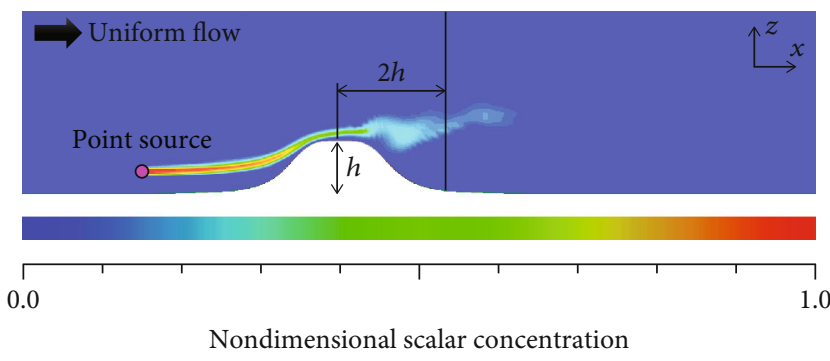

(a)

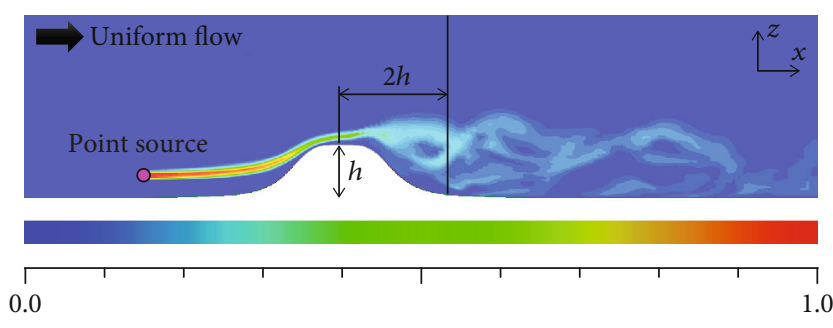

Nondimensional scalar concentration

(c)

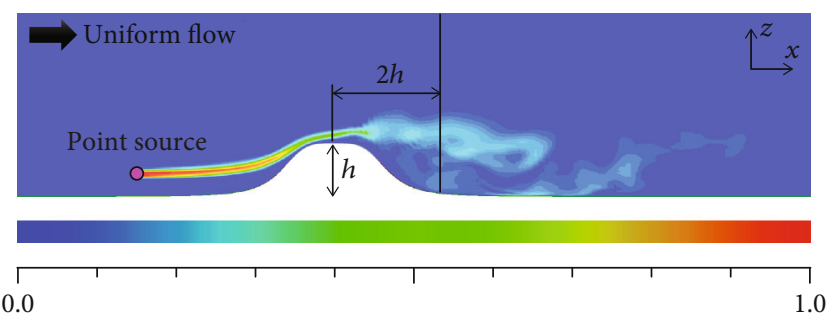

Nondimensional scalar concentration

(b)

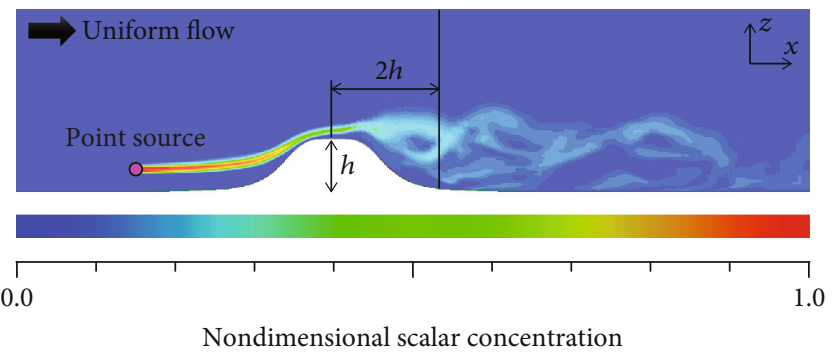

(d)

FIGURE 4: Spatial distribution of the instantaneous nondimensional scalar concentration in the $x-z$ plane under Case 1: (a) nondimensional time $t=107$; (b) $t=112$; (c) $t=117$; (d) $t=122$.

consider passive scalar transport and diffusion, the standard convection-diffusion equation for a passive scalar (equation (3)) was solved by coupling it with equation (1) and equation (2) for the atmospheric wind fields. Here, Re and Pr in equations (2) and (3) are the Reynolds number and Prandtl number $(=0.71)$, respectively. Since the present study investigated wind fields with a mean wind speed of $5-10 \mathrm{~m} / \mathrm{s}$, the effects of vertical thermal stratification of the atmosphere (atmospheric stability) were neglected.

For the computational algorithm, a method similar to the fractional step (FS) method [20] was used, and a time march- ing method based on the Adams-Bashforth two-step explicit method was adopted. Poisson's equation for pressure was solved using the successive overrelaxation (SOR) method. For the discretization of all spatial terms except for the convective term in equation (2), a second-order central difference scheme was applied. For the convective term, a third-order upwind difference scheme was applied. The interpolation technique by Kajishima [21] was used for the fourth-order central differencing that appears in the discretized form of the convective term. For the weighting of the numerical diffusion term in the convective term discretized 


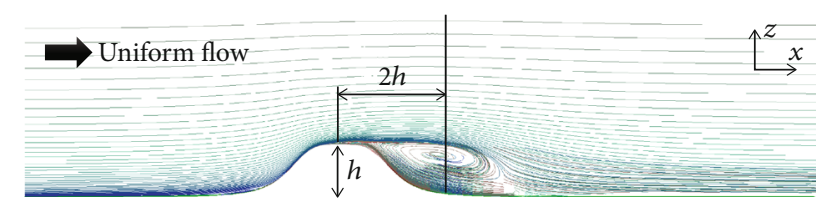

(a)

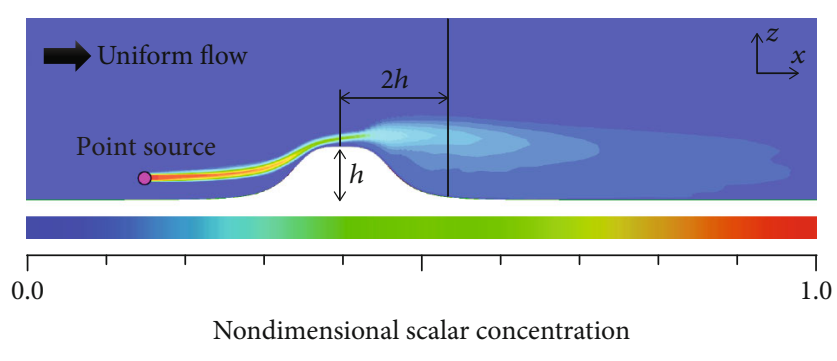

(b)

FIgure 5: Time-averaged numerical results in the $x-z$ plane under Case 1: (a) flow field by streamlines and (b) the corresponding scalar concentration field.

by third-order upwind differencing, $\alpha=0.5$ was used, as opposed to $\alpha=3.0$ from the Kawamura-Kuwahara scheme [22], to minimize the influence of numerical diffusion. For LES subgrid-scale modeling, the standard Smagorinsky model [23] was adopted with a model coefficient $\left(C_{s}\right)$ of 0.1 in conjunction with a wall-damping function (equations (4)-(12)).

$$
\begin{aligned}
& \frac{\partial \bar{u}_{i}}{\partial x_{i}}=0 \\
& \frac{\partial \bar{u}_{i}}{\partial t}+\bar{u}_{j} \frac{\partial \bar{u}_{i}}{\partial x_{j}}=-\frac{\partial \bar{p}}{\partial x_{i}}+\frac{1}{\operatorname{Re}} \frac{\partial^{2} \bar{u}_{i}}{\partial x_{j} \partial x_{j}}-\frac{\partial \tau_{i j}}{\partial x_{j}}, \\
& \frac{\partial \bar{c}}{\partial t}+\bar{u}_{j} \frac{\partial \bar{c}}{\partial x_{j}}=\frac{1}{\operatorname{RePr}} \frac{\partial^{2} \bar{c}}{\partial x_{j} \partial x_{j}}-\frac{\partial h_{j}}{\partial x_{j}}, \\
& \tau_{i j} \approx \overline{u_{i}^{\prime} u_{j}^{\prime}} \approx \frac{1}{3} \overline{u_{k}^{\prime} u_{k}^{\prime}} \delta_{i j}-2 v_{\mathrm{SGS}} \bar{S}_{i j}, \\
& v_{\mathrm{SGS}}=\left(C_{s} f_{s} \Delta\right)^{2}|\bar{S}| \text {, } \\
& |\bar{S}|=\left(2 \bar{S}_{i j} \bar{S}_{i j}\right)^{1 / 2}, \\
& \bar{S}_{i j}=\frac{1}{2}\left(\frac{\partial \bar{u}_{i}}{\partial x_{j}}+\frac{\partial \bar{u}_{j}}{\partial x_{i}}\right), \\
& f_{s}=1-\exp \left(-z^{+} / 25\right) \text {, } \\
& \Delta=\left(\Delta_{x} \cdot \Delta_{y} \cdot \Delta_{z}\right)^{1 / 3}, \\
& h_{j}=-\alpha_{\mathrm{SGS}} \frac{\partial \bar{c}}{\partial x_{j}}, \\
& \alpha_{\mathrm{SGS}}=\frac{v_{\mathrm{SGS}}}{\operatorname{Pr}_{\mathrm{SGS}}}, \\
& \operatorname{Pr}_{\mathrm{SGS}}=0.5 \text {. }
\end{aligned}
$$

\section{Validation Testing of the Gas Diffusion Modeling Implemented Using the In-House RIAM-COMPACT LES Solver}

3.1. The Case of Simple Terrain (Isolated Bell-Shaped Hill). In this study, the prediction accuracy was verified by comparing our results with the measurements of the concentration dis- tributions downwind of a point source in a wind tunnel experiment in a neutral case (see Figure 1) produced by Snyder and Hunt in 1984 [24]. Here, we consider a neutral flow passing a bell-shaped hill on a flat terrain. The bell shape of the hill is defined as follows:

$$
f(r)=\frac{1}{1+r^{4}}
$$

where $f(r)$ is the height and $r$ is the distance from the center of the hill. Here, when $r=0$, we define $f(0)=h$ (maximum height of the hill). The computational domain has a space of $15 h$ in the streamwise direction $(x), 10 h$ in the spanwise direction $(y)$, and $9 h$ in the vertical direction $(z)$. The center of the hill is located $6 h$ downstream of the inflow boundary. The number of computational grid points is $151(x) \times 101(y$ )$\times 61(z)$ points, and the grid resolution near the ground surface and around the terrain is fine. The grid width in each direction is $\Delta x=\Delta y=0.1 \mathrm{~h}$ (uniform grid width) and $\Delta z=$ $3.5 \times 10^{-3}-6.0 \times 10^{-1} h$. In this research, various sensitive analyses were performed for the horizontal spatial grid resolution and the length-of-time averaging. These parameters are shown in Table 1. The computational grid used in this study is shown in Figure 1.

To perform validation testing of the gas diffusion modeling, the tracer gas release started from the nondimensional time $t=100$, when the flow field that formed around the isolated hill was fully developed (see Figures 2 and 3). A closer look at Figure 3 shows that the flow separates near the top of the hill, and the separated shear layer rolls up into an isolated vortex. The isolated vortices form large-scale vortices, which are shed almost periodically to the downstream side of the hill. At each time step, the maximum concentration value of the tracer gas $(C=1.0)$ is given at the point source, and continuous tracer gas emissions are reproduced, as shown in Figure 4. The location of the stack was 3.6h upwind of the center of the polynomial hill. The boundary conditions were simulated using the conditions of the wind tunnel experiment by Snyder and Hunt [24] (quoted for accuracy verification). In the wind tunnel experiment, to avoid the influence of the boundary layer generated on the floor surface of the wind tunnel, the model was installed near the inlet of the test section to minimize the influence of the boundary layer thickness. To accurately simulate the real situation of the wind tunnel experiment, a uniform flow was given at the inflow boundary in the numerical simulation. The 


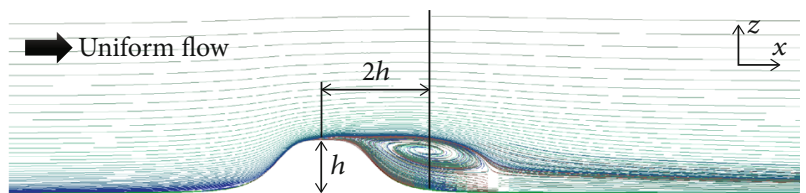

(a)

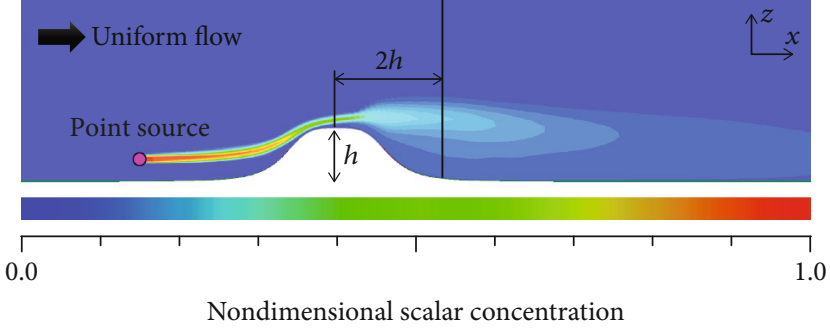

(b)

Figure 6: Time-averaged numerical results in the $x-z$ plane under Case 2: (a) flow field by streamlines and (b) the corresponding scalar concentration field.

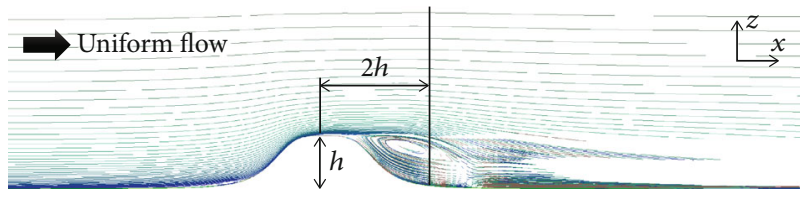

(a)

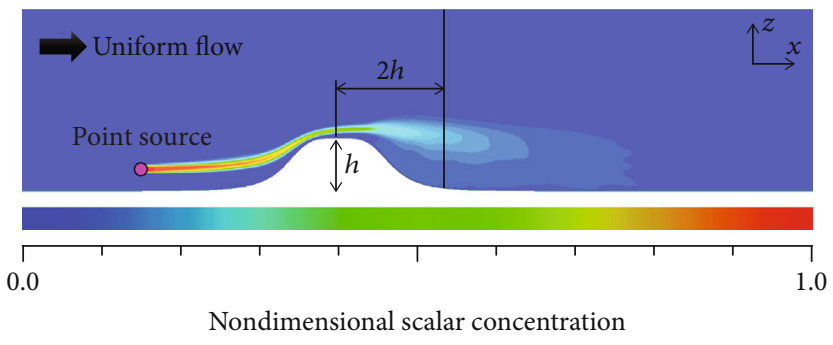

(b)

Figure 7: Time-averaged numerical results in the $x-z$ plane under Case 3: (a) flow field by streamlines and (b) the corresponding scalar concentration field.

Reynolds number defined by the height, $h$, of the isolated hill and the uniform flow, $U$, was set to $1.0 \times 10^{4}$, which is almost the same as the one used in the wind tunnel experiment $\left(4.5 \times 10^{4}\right)$. Again, Figure 4 corresponds to Figure 3. Turbulent entrainment phenomenon of the scalar concentration in the wake region behind the hill can be clearly observed.

In this research, various sensitive analyses were performed for the horizontal spatial grid resolution and the length-of-time averages, as shown in Table 1. First, using Figures 5 and 6, we show the results of comparing the effects of the length-of-time average based on the calculation results with the same horizontal grid resolution. Here, as shown in Table 1, the time-averaged length of Case 2 was 10 times that of Case 1. Focusing on the time-averaged flow fields shown in Figure 5(a) and Figure 6(a), it can be seen that the size of the vortex region formed behind the hill and the position of the vortex center were almost the same in both cases. Along with these numerical results, the time-averaged scalar concentration fields corresponding to Figure 5(a) and Figure 6(a) also obtained almost the same results in both cases (see Figure 5(b) and Figure 6(b)). Next, using Figures 5 and 7, we examined the effect of the horizontal grid resolution. As shown in Table 1 and Figure 1, the horizontal grid resolution in Case 3 was half that of Case 1 and Case 2. As a result, we also set Case 3 to half the nondimensional time increment of Case 1 and Case 2. The grid resolution in the vertical direction was the same in both cases. Furthermore, the length-oftime averaging was the same in both cases. Focusing on the time-averaged flow fields, Figure 5(a) and Figure 7(a) showed that although there was a slight difference in the central position of the vortex region formed behind the hill, its size was almost the same. As a result, no significant difference was

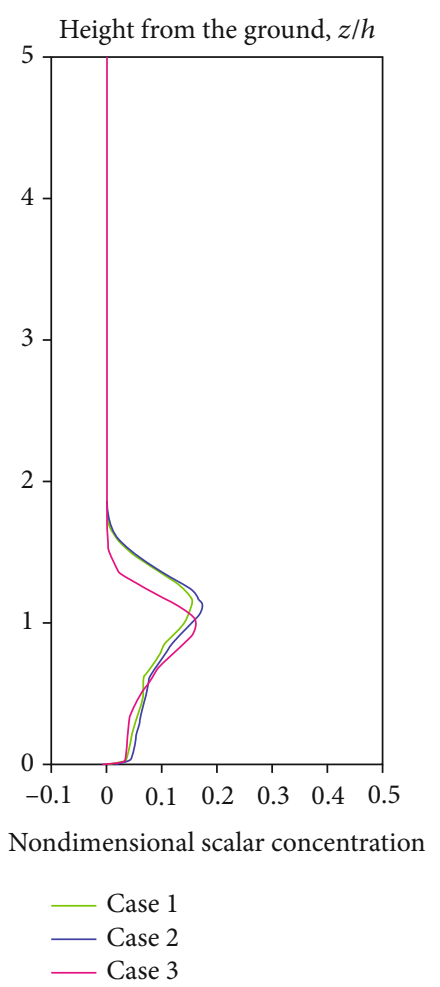

Figure 8: Vertical distribution of the scalar concentration at position $2 h$ downstream of the hill center extracted from the timeaveraged field. Here, $h$ indicates the hill height.

found in the corresponding time-averaged scalar concentration field. Figure 8 shows the vertical distribution of the scalar concentration at position $2 h$ downstream of the hill center 


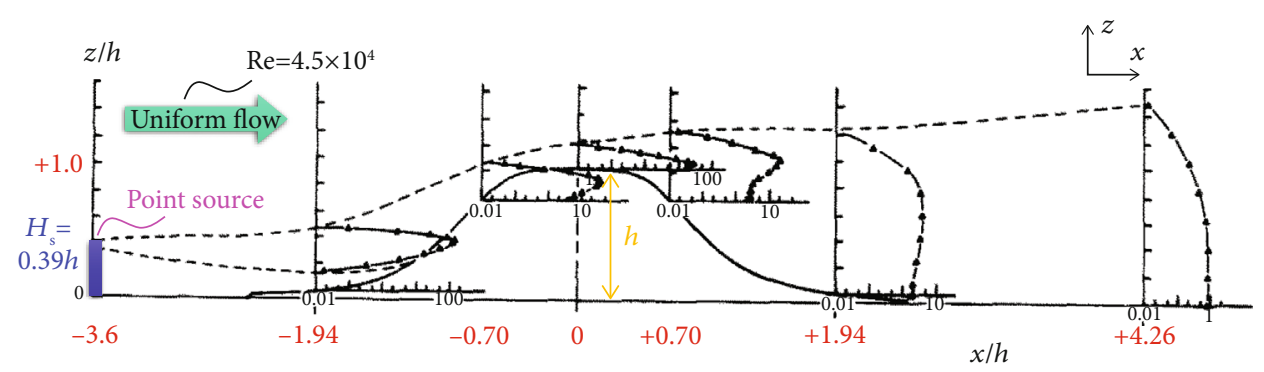

FIGURE 9: Measurements of the time-averaged concentration distributions downwind of a point source in the wind tunnel experiment for a neutral case [24].

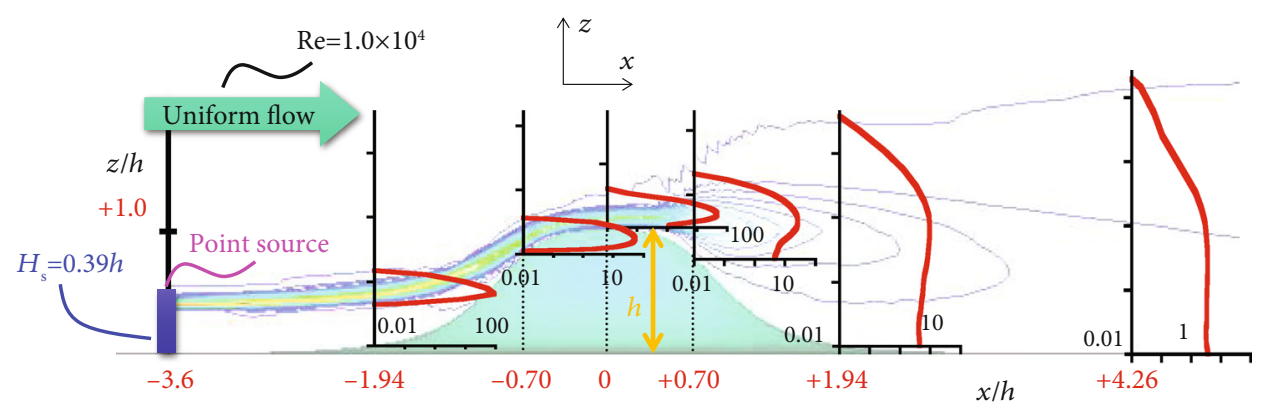

FIGURE 10: Measurements of the time-averaged concentration distributions downwind of a point source in the numerical simulation for a neutral case (Case 1).
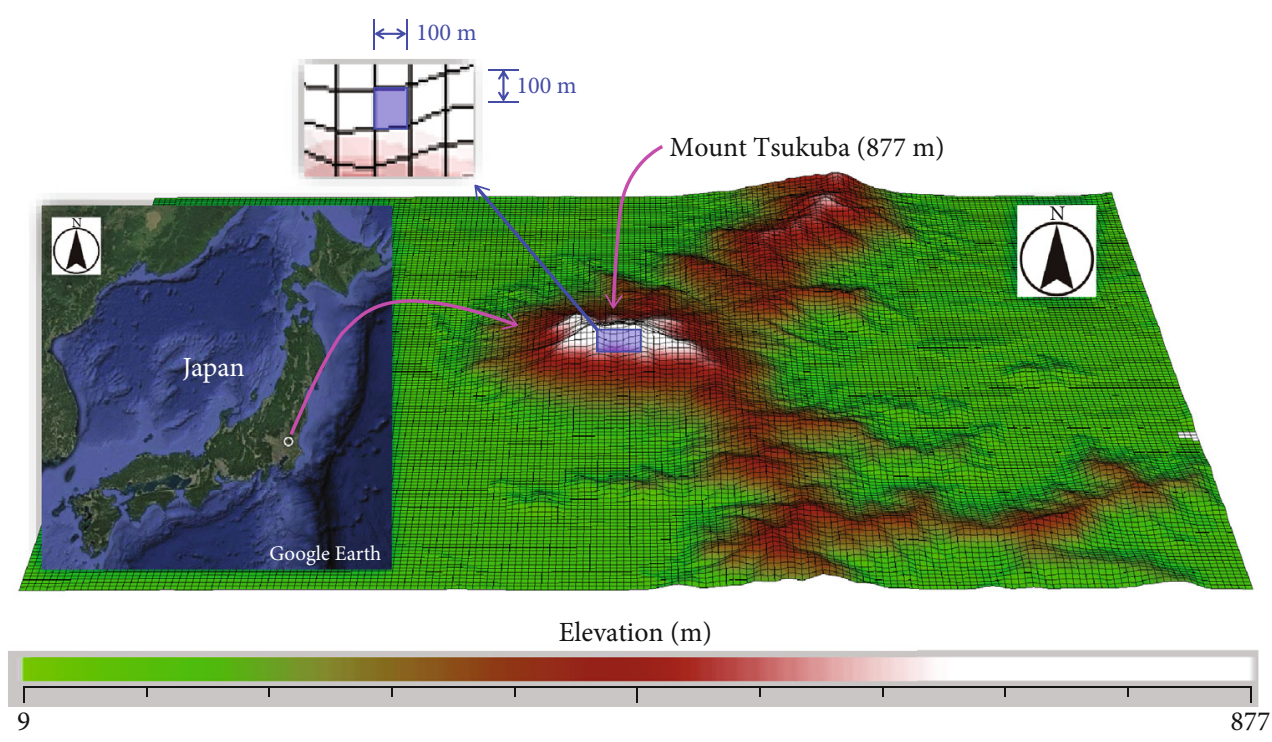

FIGURE 11: Mt. Tsukuba targeted in this study.

displayed in Figures 3 to 7. Case 1 and Case 2 displayed almost the same behavior for all ranges in the vertical direction. In Case 3, the height of the maximum concentration was slightly lower than that in Case 1 and Case 2, but there was no significant difference in the maximum value itself.

Finally, a comparison of the numerical results obtained in this study with the measurements of the concentration distributions in the wind tunnel experiment for a neutral case by Snyder and Hunt [24] is provided. Figure 9 shows the results of their wind tunnel experiment. As already presented above, the plumes impacted the front surface of the hill and moved over the hilltop. Within and near the wake, turbulence that has significant effects on the diffusion chimney plumes was generated. Turbulent mixing dramatically reduced the concentration of the plumes. Furthermore, large-scale vortex shedding in the wake or flow meander can significantly decrease the average concentration. Figure 10 shows the simulation results corresponding to Figure 9. Here, the 


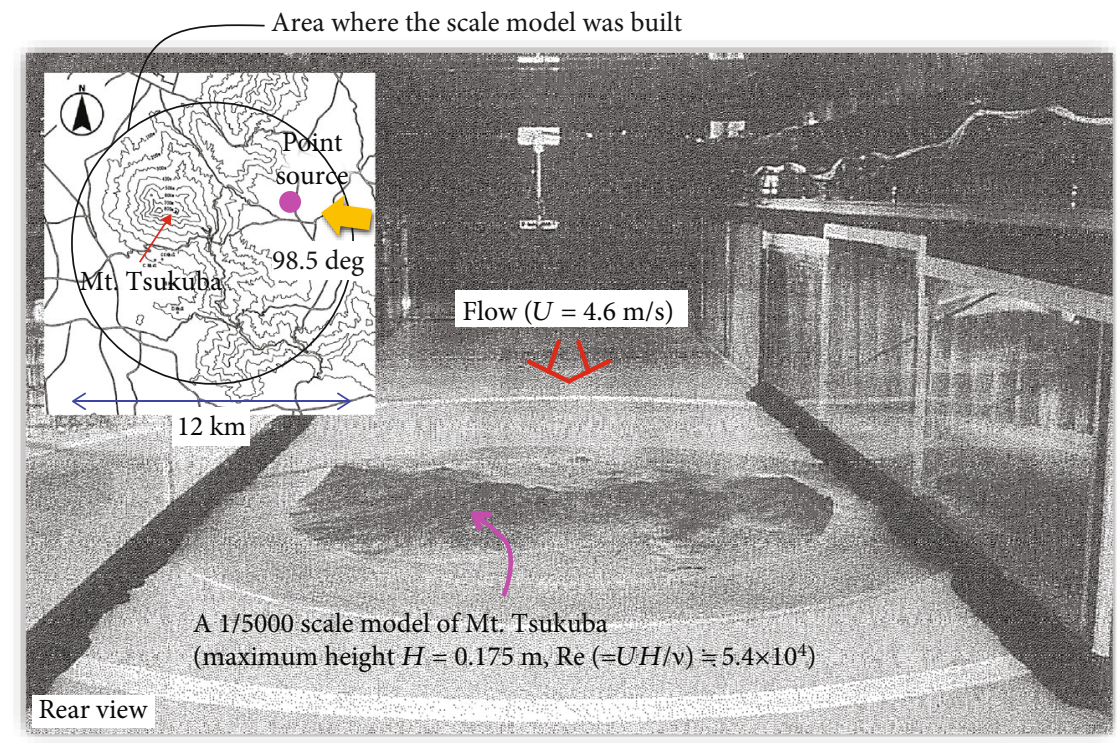

Figure 12: Scale model of Mt. Tsukuba installed in the wind tunnel [25].

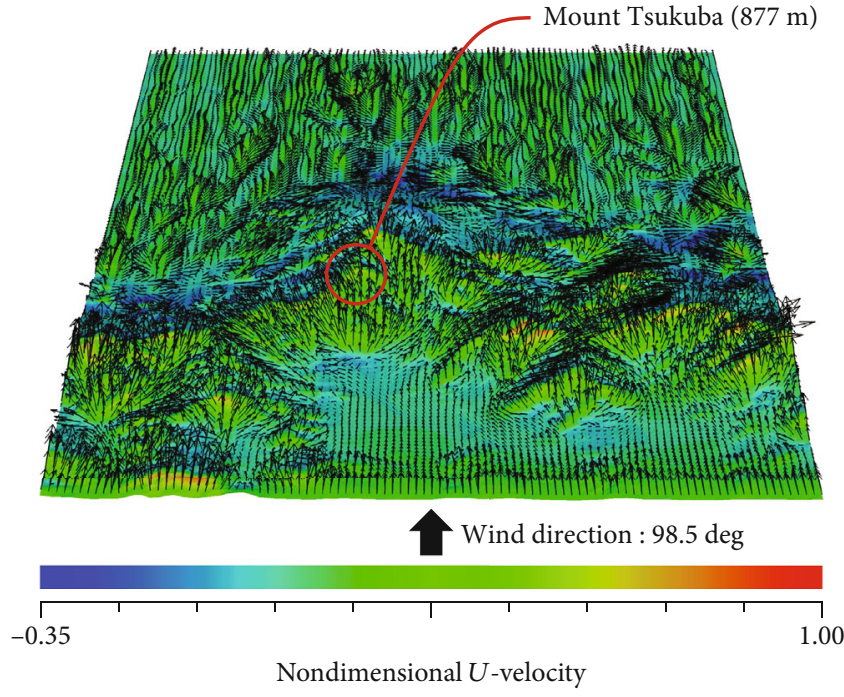

(a)

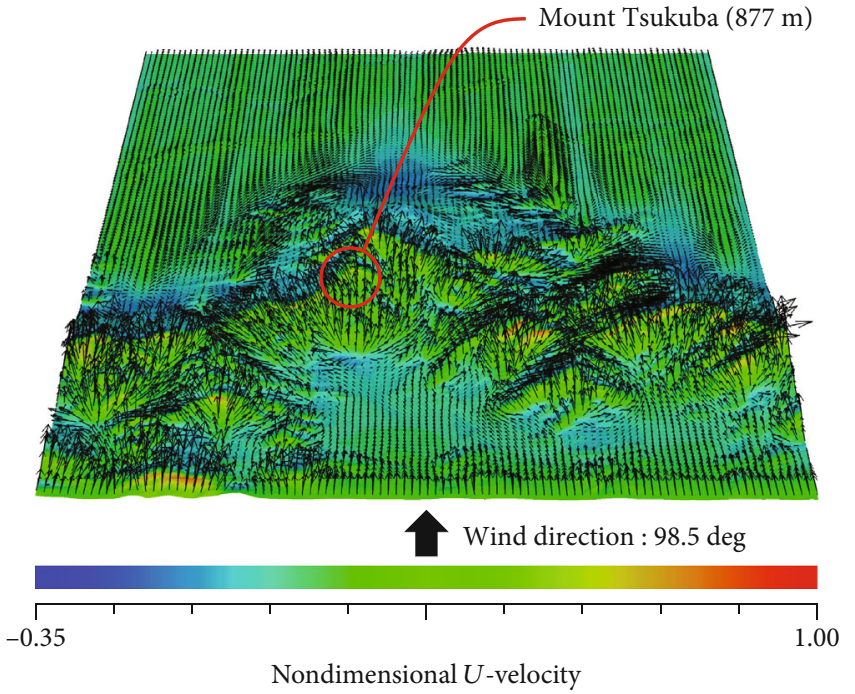

(b)

FIGURE 13: Flow pattern near the terrain surface $(z=8 \mathrm{~m})$ : (a) instantaneous flow field and (b) time-averaged flow field.

concentration distributions were compared by matching the following dimensionless concentrations using a numerical simulation and wind tunnel experiment:

$$
\frac{U C h^{2}}{Q}
$$

where $U$ is the uniform flow speed, $h$ is the height of the hill, $C$ is the concentration, and $Q$ is the emission amount. When comparing the results of both numerical simulation and wind tunnel experiment, the concentration distributions were shown to be in good agreement, both qualitatively and quantitatively, on both the upstream and downstream sides of the isolated hill.
3.2. The Case of Complex Terrain (Mount Tsukuba). For the case of complex terrain, the prediction accuracy of the numerical simulation was verified by comparing it with the result of the wind tunnel experiment for turbulent diffusion in complex terrain reported by Hayashi et al. in 2001 [25]. The topography targeted in this study was Mt. Tsukuba, Japan (see Figure 11), which features relatively simple and isolated topography.

In particular, we paid attention to how the central axis of the plume released from an upwind point of Mt. Tsukuba is directly affected by the topographic effects. The wind tunnel experiment was conducted using the diffusion wind tunnel equipment owned by Mitsubishi Heavy Industries (MHI). The test section of the wind tunnel used was $25 \mathrm{~m}$ long, $3 \mathrm{~m}$ wide, and $2 \mathrm{~m}$ high. A 1/5000 scale model was installed in 


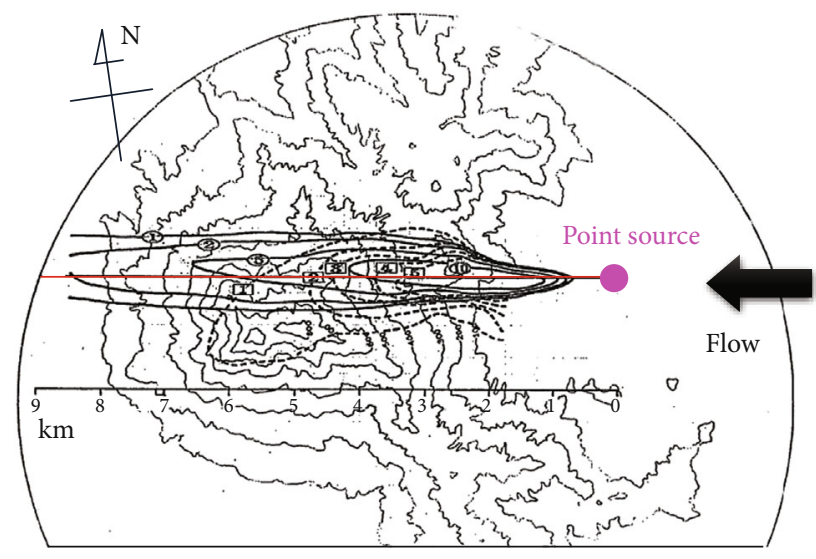

Figure 14: Measurements of the average surface concentration contours on Mt. Tsukuba in the wind tunnel experiment for a neutral case [25].

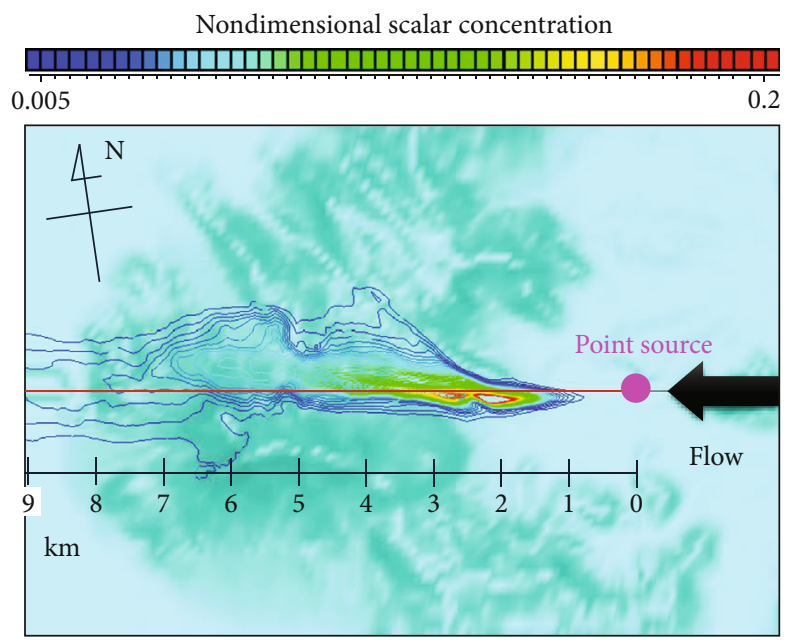

Figure 15: Measurements of the average surface concentration contours on Mt. Tsukuba in the numerical simulation for a neutral case.

the wind tunnel. Therefore, in the wind tunnel, Mt. Tsukuba was reproduced at a maximum height of $0.175 \mathrm{~m}$. The blockage ratio defined by the wind tunnel height and the model maximum height was $8.75 \%$. Figure 12 shows the scale model installed in the wind tunnel. The vertical distribution of wind velocity was reproduced by adjusting the airflow to obtain a boundary layer thickness of $\delta=0.8 \mathrm{~m}$ and a power index of $n=1 / 7$. The target wind direction was $98.5^{\circ}$, as shown in Figure 12.

Next, the numerical simulation carried out in this research is outlined. The numerical simulation was performed under almost the same conditions as the wind tunnel experiment. The computational domain was $15 \mathrm{~km}$ in the streamwise direction $(x), 10 \mathrm{~km}$ in the spanwise direction $(y)$, and $4 \mathrm{~km}$ in the vertical direction $(z)$. The shape of the terrain was created based on the $10 \mathrm{~m}$ digital elevation data of the Geospatial Information Authority of Japan. The number of grid points was $151(x) \times 101(y) \times 61(z)$. The grid resolution in the horizontal direction was $100 \mathrm{~m}$, as shown in Figure 11. The vertical direction was set close to the ground surface, and the minimum grid width was $2.5 \mathrm{~m}$.
We considered the flow pattern near the terrain surface $(z=8 \mathrm{~m})$ obtained from the numerical simulation shown in Figure 13. In Figure 13, the flow field is visualized using the velocity vectors and distributions of the nondimensional $U$ -velocity. In both the instantaneous and time-averaged flow fields, the complex flow fields, such as flow impingement, flow separation, and local flow acceleration, can be confirmed near the terrain surface. In particular, the area around Mt. Tsukuba surrounded by the solid line in Figure 13 flowed over and around the mountains and through the valleys between the mountains. In this way, the present study successfully reproduced the topographic effect on the wind around Mt. Tsukuba.

Finally, a comparison of the numerical simulation results obtained in this study and the wind tunnel experiment conducted by Hayashi et al. in 2001 [25] is provided. Figure 14 shows the measurements of the average surface concentration contours of Mt. Tsukuba in the wind tunnel experiment for a neutral case. Figure 15 shows the measurements of the average surface concentration contours on Mt. Tsukuba in the numerical simulation for a neutral case. Similar to the simulation of the isolated hill, the tracer gas release started 


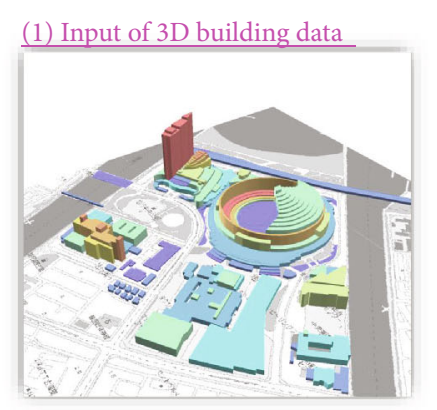

(3) Flow calculation
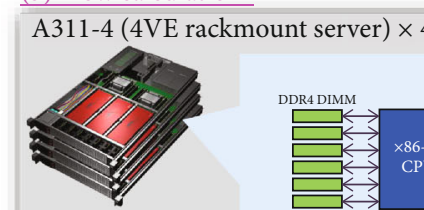

ount server) $\times 4$

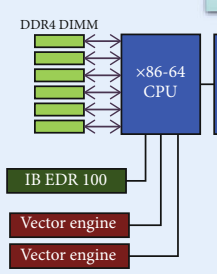

NEC SX-Aurora TSUBASA A311-4

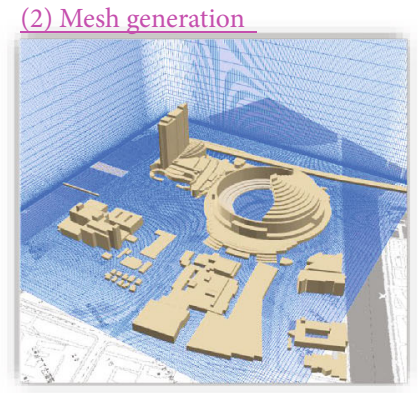

High performance computational fluid dynamics (CFD) on vector-parallel supercomputer

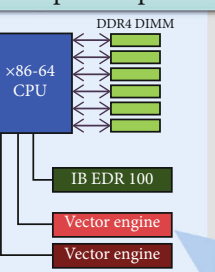

Vector engine : type 10BE

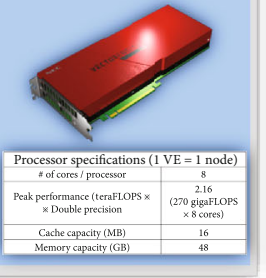

$(1,2)$ CFD preprocessing

(3) CFD solver

(4) CFD postprocessing

FIGURE 16: Procedure employed for the computational fluid dynamics (CFD) simulation in the present study.

from the nondimensional time $t=100$. The time-averaging length was the same as that of the hill. Both patterns of the average surface concentration contours were shown to be very similar. Due to the flow pattern near the terrain surface described in Figure 13, it became clear that the central axis of the plume released from the upwind point of Mt. Tsukuba was directly affected.

\section{Application Testing of the Gas Diffusion Modeling Implemented in the In-House Airflow Analyst LES Solver}

Next, using the latest detailed urban data, the Japan National Stadium (Tokyo Olympic Stadium) was reproduced in detail, and a CFD simulation with a large number of grid points/cells was performed. This section introduces the results obtained from these large-scale computations.

4.1. Structured Mesh Generation Based on the GIS Technique and Numerical Conditions. Generally, when performing CFD simulations for urban areas, the four processes shown in Figure 16 are required. The work of inputting the $3 \mathrm{D}$ building data shown in Figure 16 required a large amount of time. Here, we outline the 3D modeling method for the Japan National Stadium, which was the subject of this study.

AW3D [18] was used for the data representing the current topography, buildings, and the position and height of trees. AW3D [18] combines city images captured by various US high-resolution satellites from various angles without blind spots. AW3D is a type of 3D map created using technology that can identify buildings and trees one dimensionally. For a building with a nonhorizontal roof (top surface), changes in height are individually captured, allowing a detailed urban shape to be reproduced. AW3D covers the whole world and is characterized because it can even be used in areas where it is difficult to obtain base map data. The provided data are divided into a digital terrain model (DTM) showing the surface elevation with a $5 \mathrm{~m}$ resolution, a building polygon showing the building shape, and a tree polygon showing the tree shape. All are provided in the GIS file format (TIFF format and ESRI shape format) in the Universal Transverse Mercator (UTM) coordinate system. These data groups can be directly used for numerical wind simulations without any special processing work. 


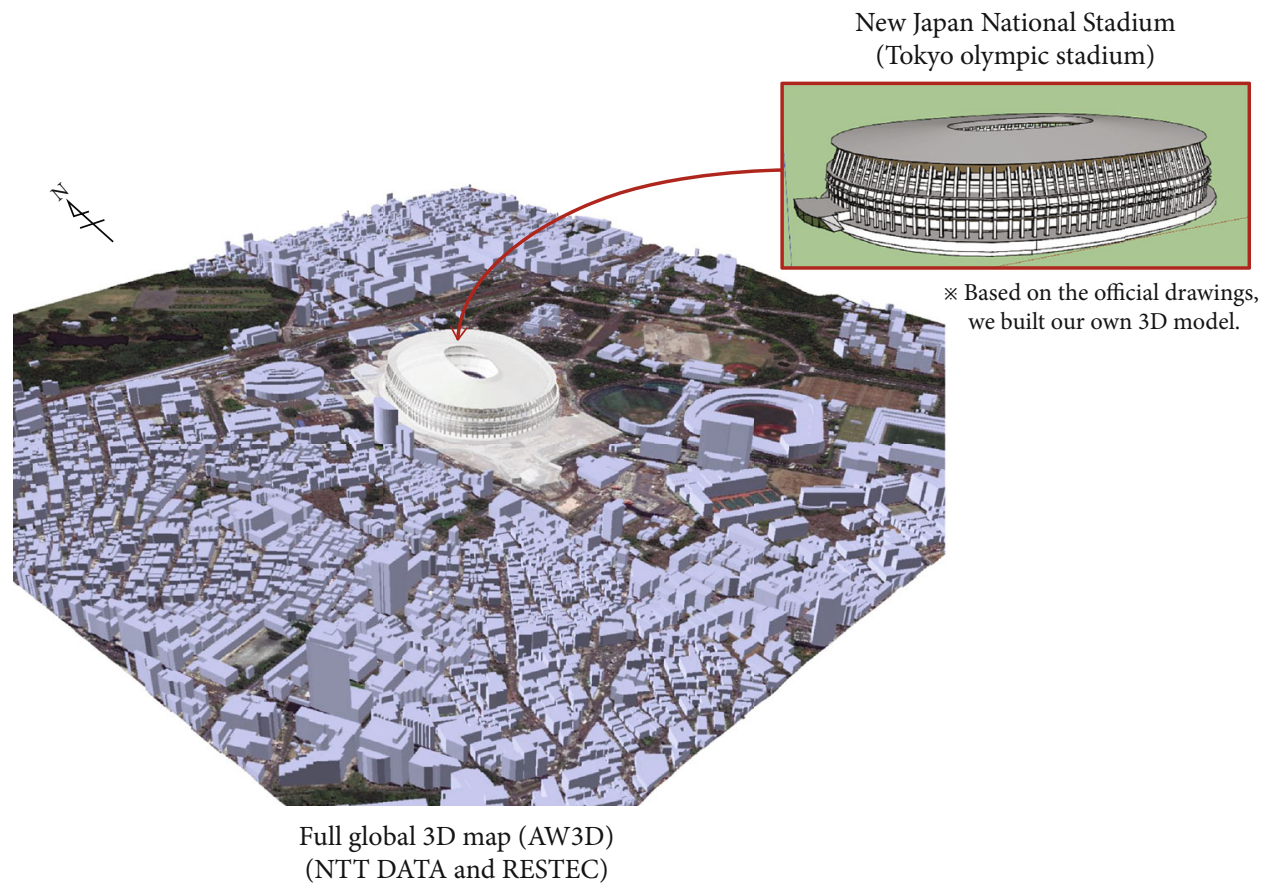

FIgURE 17: Japan National Stadium 3D model created based on Advanced World 3D Map (AW3D) and surrounding 3D models.

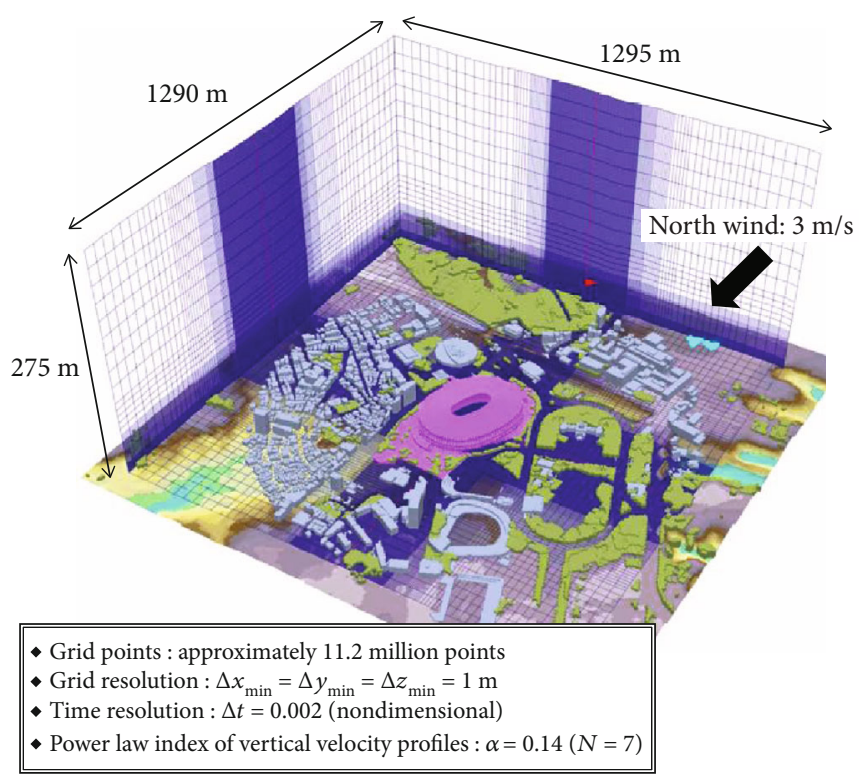

Figure 18: Computational domain and grid information.

In parallel, considering the building shape of the Japan National Stadium, 3D data were created independently using the $3 \mathrm{D}$ modeling software SketchUP based on the publicly available plan. The created 3D model data were imported into GIS according to the position and scale of the UTM coordinate system. Airflow Analyst can recognize 3D model data as DTM image (in the TIFF format), an ESRI shapefile, or CAD and BIM files. In this way, the user (planner) can easily generate the grid for CFD calculations. As a result, the planner is freed from CFD preprocessing and can concentrate on considering the simulation results and revising the plan based on them. Figure 17 shows the state of the Japan

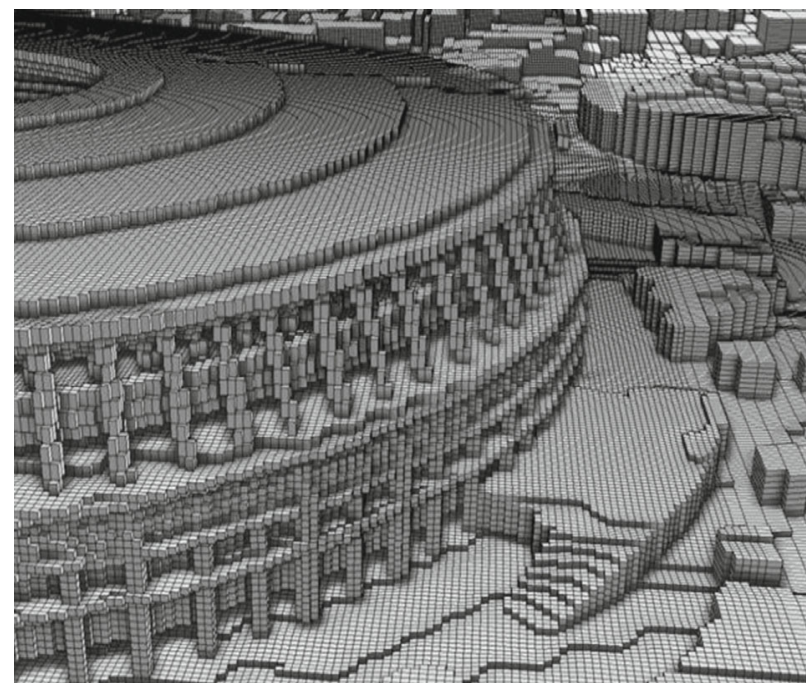

FIgURE 19: Enlarged view of the grid around the Japan National Stadium.

National Stadium, which was the subject of this research, and its surroundings as a computer reproduction. In this research, a numerical wind simulation (CFD simulation) was only conducted for the Japan National Stadium, without considering the surrounding topography, buildings, and surface roughness values. Figure 18 shows the computational domain area and other parameters set in this study. Figure 19 presents an enlarged view of the computational grid around the Japan National Stadium.

4.2. Numerical Results and Discussions. First, we consider the structure of the flow pattern inside the Japan National 


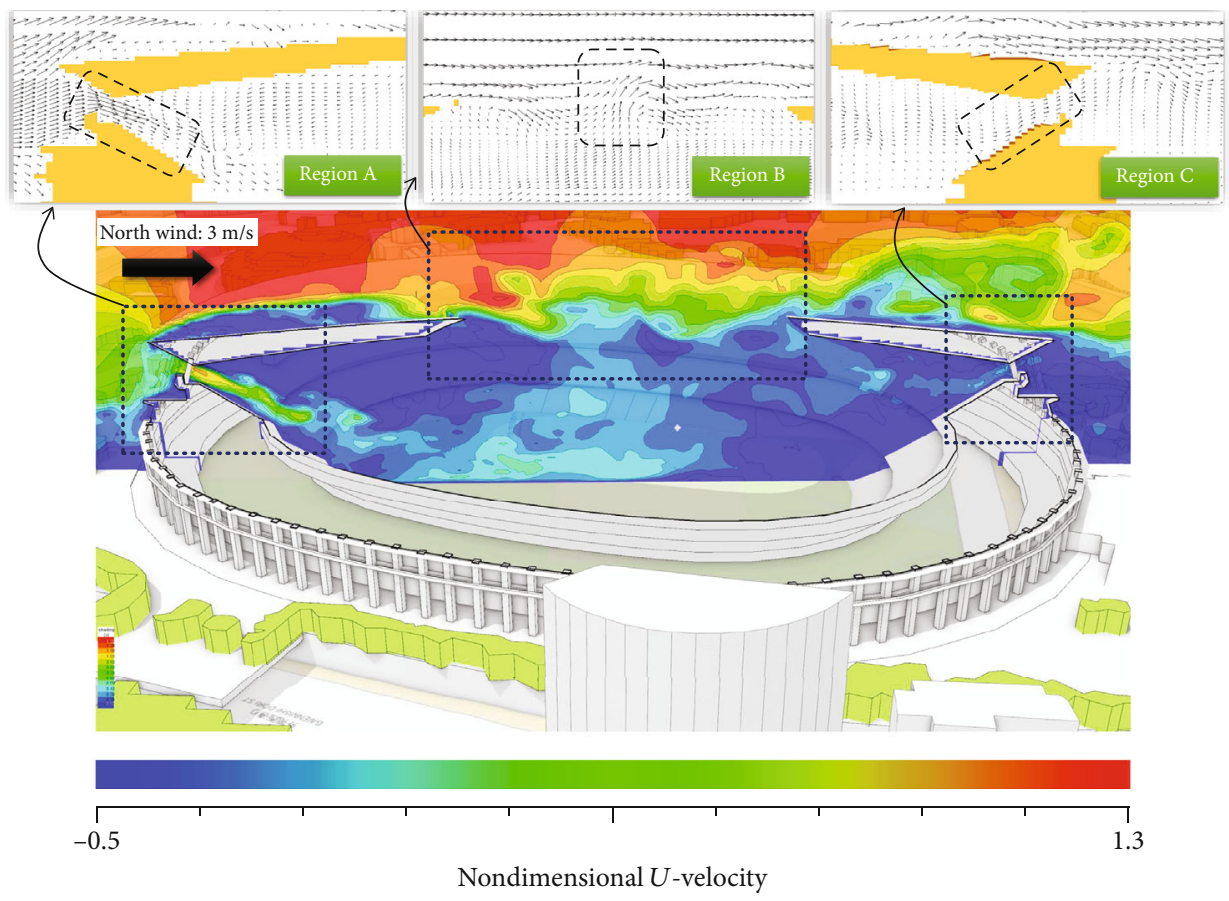

(a)

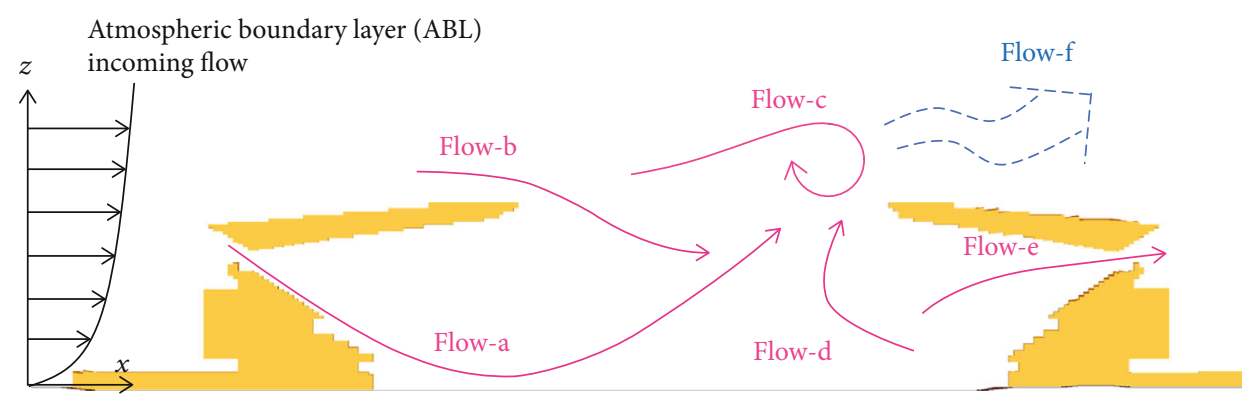

(b)

FIgURe 20: Flow visualization and structure inside and around the Japan National Stadium (Tokyo Olympic Stadium) for the instantaneous flow field: (a) nondimensional $U$-velocity and vectors and (b) a schematic view.

Stadium. As shown in Figure 18, the present study targeted the north wind. The wind speed was set to $3 \mathrm{~m} / \mathrm{s}$ at $40 \mathrm{~m}$ above the ground. Figure 20 shows the flow visualization and structure inside the Japan National Stadium for the instantaneous flow field. Figure 20(a) shows the nondimensional $U$-velocity and vectors. Next, we explain the features of the structure of the flow pattern inside the Japan National Stadium by dividing it into three main areas. Focusing on region $\mathrm{A}$, it can be seen that the wind outside the Japan National Stadium entered the inside of the stadium. Notably, the wind from outside the stadium accelerated locally and blew downward near the ground. The wind that blew downward near the ground blew out of the stadium again in region $B$. In region $B$, the separated flow from the roof of the stadium played an important role in the mechanism by which the wind inside the stadium blew out of the stadium. In other words, the pressure in the separated flow region that formed from the roof dropped locally and rapidly, and the wind inside the stadium entrained to the outside of the stadium due to this phenomenon. Most of the wind inside the sta- dium blew outside the stadium in region $\mathrm{B}$. Additionally, in region $\mathrm{C}$, it can be clearly observed that some of the wind inside the stadium blew outside the stadium.

We created a nondimensional $U$-velocity and vector animation (shown in Figure 20(a)) and observed the animation in detail. As a result, the characteristics of the flow pattern inside and around the stadium (as shown in Figure 20(b)) were organized into a schematic view. As mentioned earlier, the wind that entered the inside of the stadium from the open space on the upstream side blew down to near the ground and then flowed upward (see Flow-a in Figure 20(b)). In addition, part of the wind inside the stadium exited the stadium from the open space on the downstream side. As shown in Flow-b (Figure 20), the wind outside the stadium entered from the large open space on the roof of the stadium. However, most of the wind inside the stadium, including Flow-a and Flow$\mathrm{d}$, exited the stadium from this large open space. The lowpressure portion of the vortex region that formed near the roof of the stadium shown in Flow-c pulled the wind inside the stadium further to the outside of the stadium due to the 


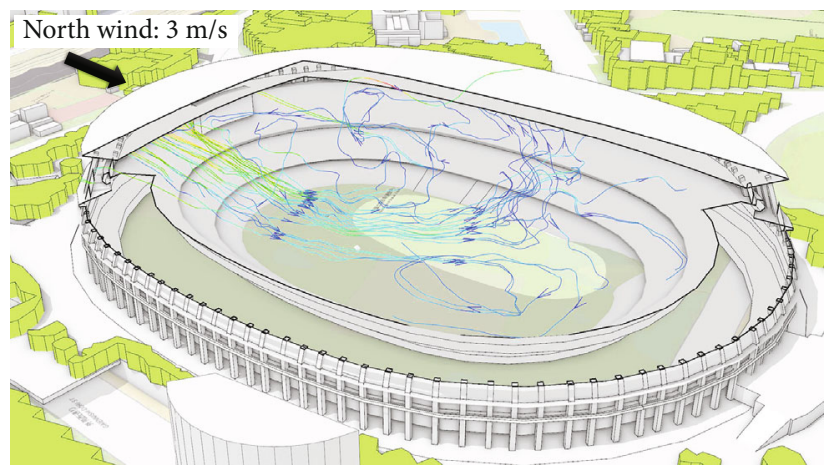

FIGURE 21: Three-dimensional flow visualization and structure inside the Japan National Stadium (Tokyo Olympic Stadium) used to study the instantaneous flow field using streamlines.

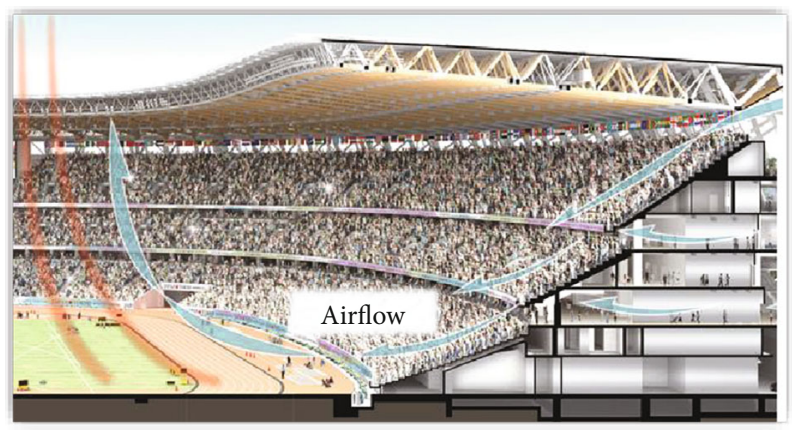

FIGURE 22: Image of the stadium cross section [26].

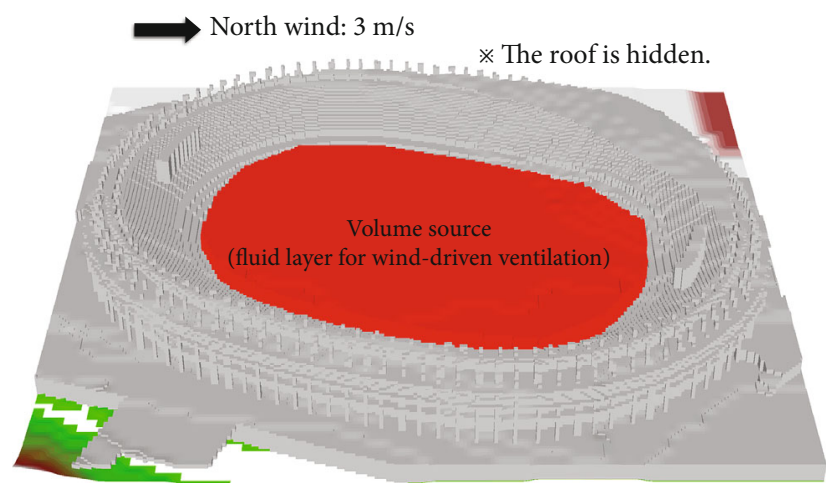

FIGURE 23: Volume source inside the stadium.

entrainment phenomenon. Figure 21 shows a 3D flow visualization of the structure inside the Japan National Stadium used to study the instantaneous flow field using streamlines. By observing these streamlines, it can be clearly observed that the wind that flowed into the stadium from upstream reached the ground. Furthermore, it can be seen that the wind inside the stadium flowed three-dimensionally in the stadium in the horizontal and vertical directions. As described above, the numerical results of this study accurately reproduced the flow pattern shown in Figure 22.

Next, we tried to reproduce the hypothetical spread and dissipation of the fluid layer (assuming the fluid layer contains COVID-19 particles) inside and outside the Japan National Stadium. We paid special attention to the effect of ventilation driven by natural wind. First, we will explore the case inside the stadium. Figure 23 shows the volume source (assuming the volume source contains COVID-19 particles) inside the stadium. In this research, we set a situation where the volume source exists on the entire ground as the initial condition. Figure 24 shows the time variation of the scalar concentration field in the $x-z$ plane. Figure 24(a) illustrates the state $1.33 \mathrm{sec}$ onds after the calculation of the scalar concentration field. In the scalar concentration field, almost no wind was ventilated, and most remained near the ground. In Figure 24(b), as explained in Figure 20, the scalar concentration that clearly entrained in the vortex region formed near the roof of the stadium and was emitted to the outside of the stadium. The effect of natural ventilation seen in Figure 24(b) is remarkable. The 


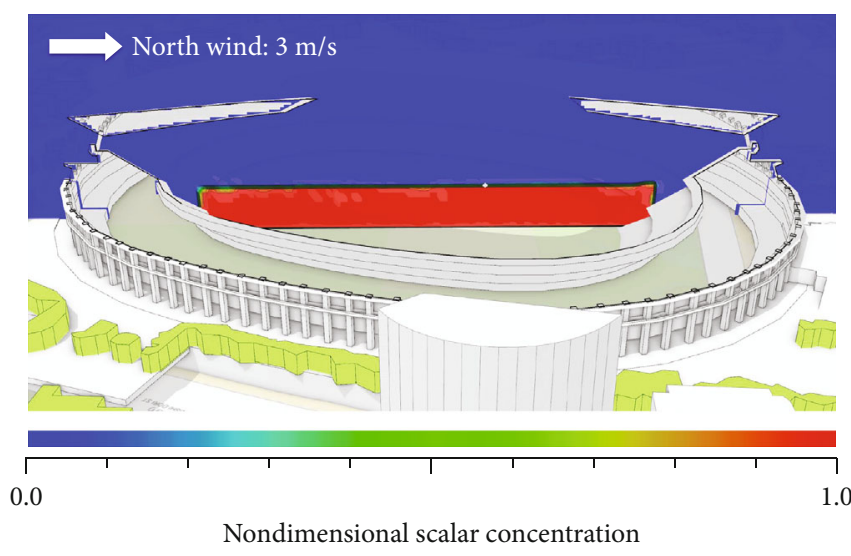

(a)

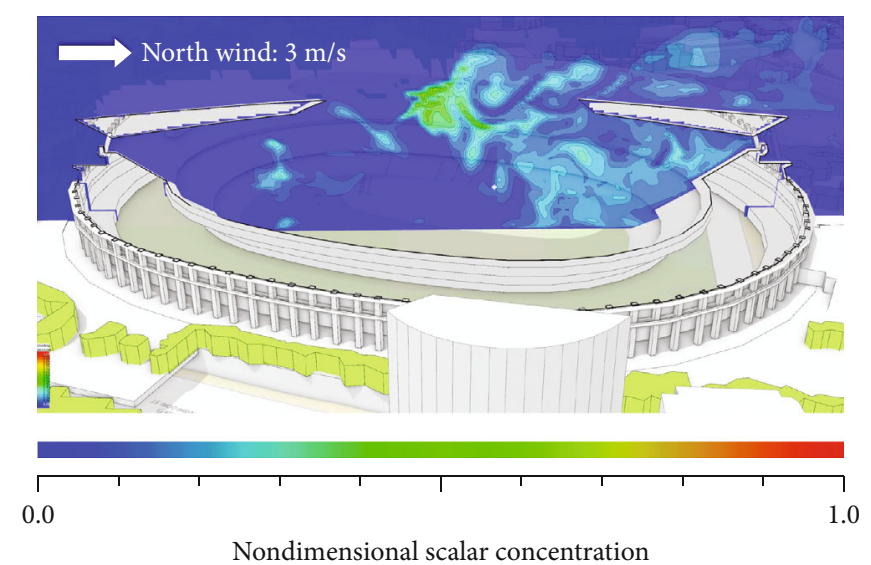

(b)

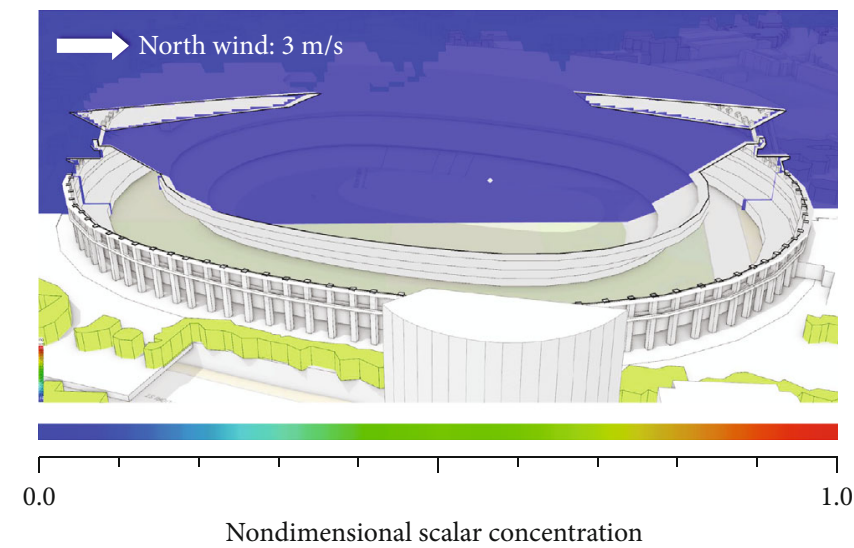

(c)

FIgURE 24: Time variation of the scalar concentration field in the $x$ - $z$ plane: (a) 1.33 seconds, (b) 354.67 seconds, and (c) 1333.33 seconds after the calculation of the scalar concentration field.

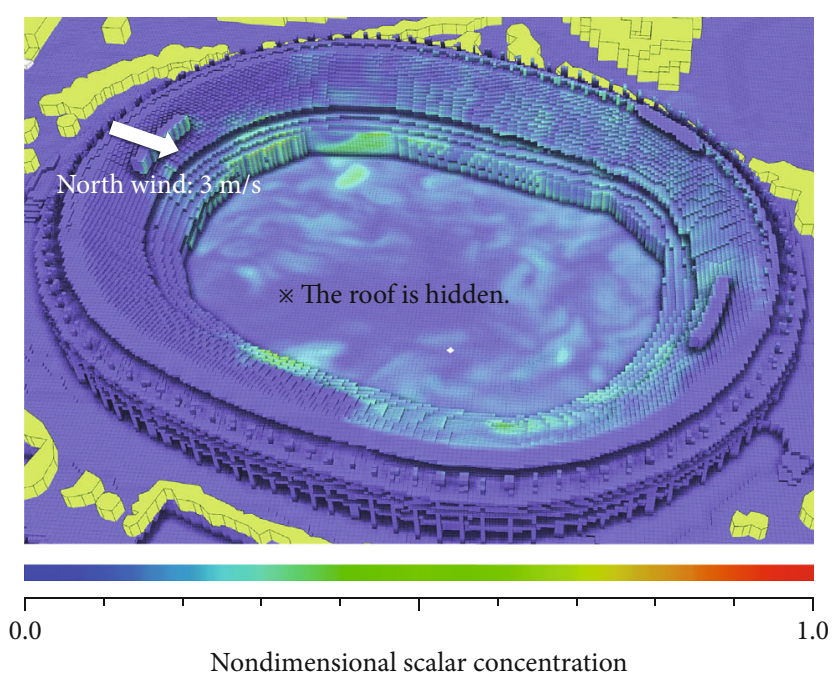

FIGURE 25: Scalar concentration distribution near the spectator seats and the ground in the stadium corresponding to Figure 24(b).

scalar concentration in the stadium was almost zero at 1333.33 seconds after the start of the scalar concentration calculation, as shown in Figure 24(c). Figure 25 shows the scalar concentration distribution near the spectator seats and the ground in the stadium corresponding to Figure 24(b). Examining this figure in detail shows that the scalar concentration was not uniformly high for all spectators; that is, there were many regions with a very low scalar density. Therefore, it is suggested that many preliminary simulations, such as those conducted in this study, be performed and that efficient 


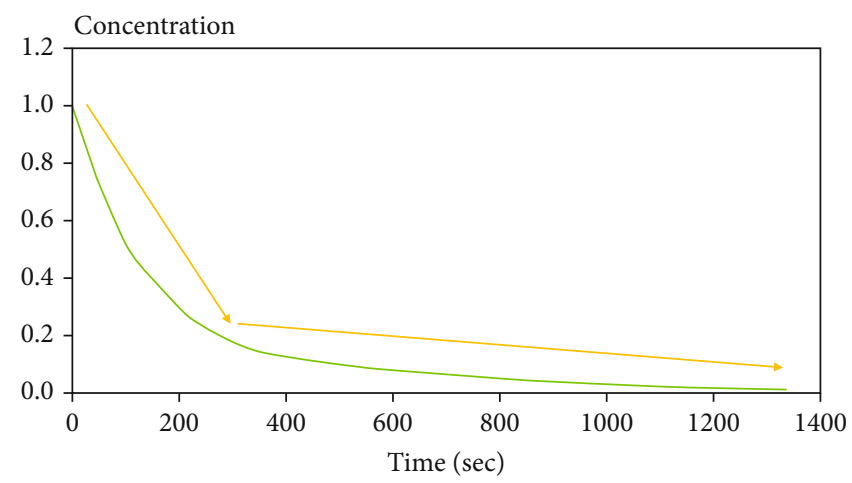

FIgURE 26: Time variation of the integrated value of the scalar concentration in the fluid layer shown in red in Figure 23.

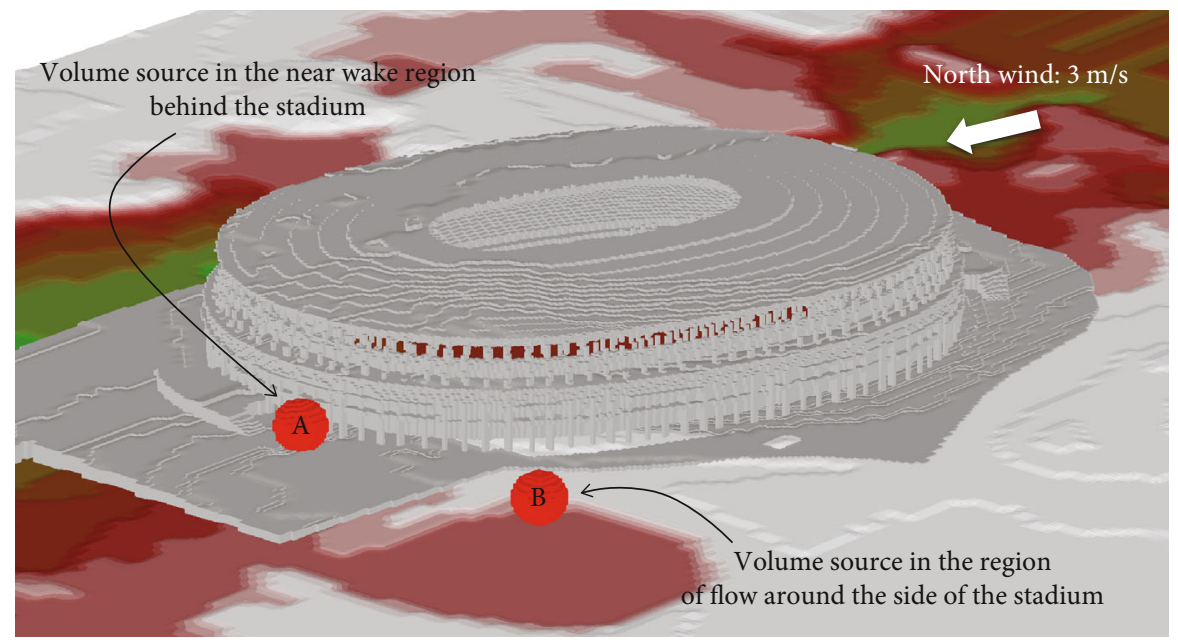

Figure 27: Two volume sources outside the Japan National Stadium (Tokyo Olympic Stadium).

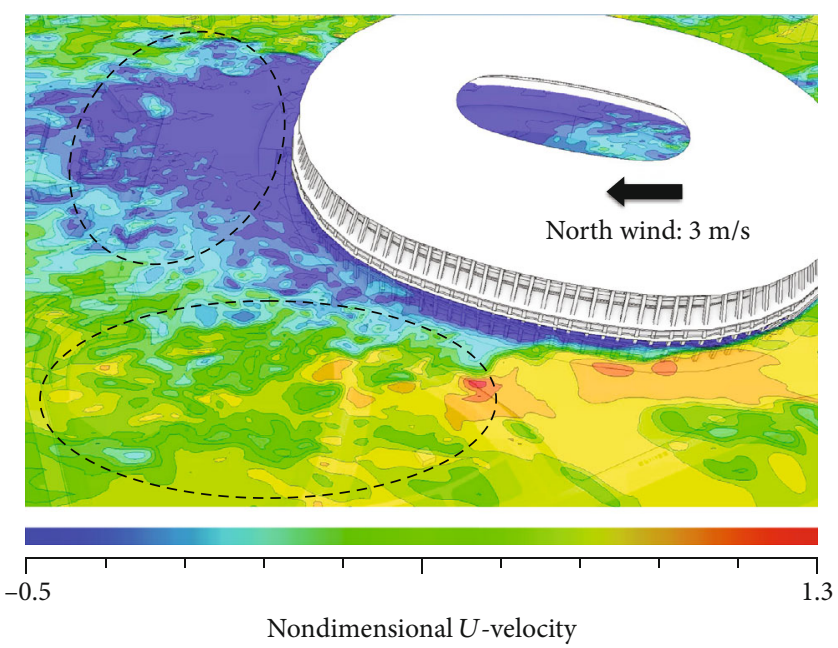

FIGURE 28: Spatial distribution of the instantaneous nondimensional $U$-velocity near the ground.

mobilization of the audience be made possible (based on these numerical results). Figure 26 shows the time variation of the integrated value of the scalar concentration in the fluid layer illustrated in red in Figure 23. In the figure, the numerical values are normalized by the initial value. It should be noted that the accumulated value rapidly decreased about $300 \mathrm{sec}-$ onds after the release of the scalar concentration. It was ultimately revealed that ventilation driven by natural wind is very effective against the hypothetical spread and dissipation of the fluid layer (assuming the fluid layer contains COVID19 particles) inside the Japan National Stadium (Tokyo Olympic Stadium). 


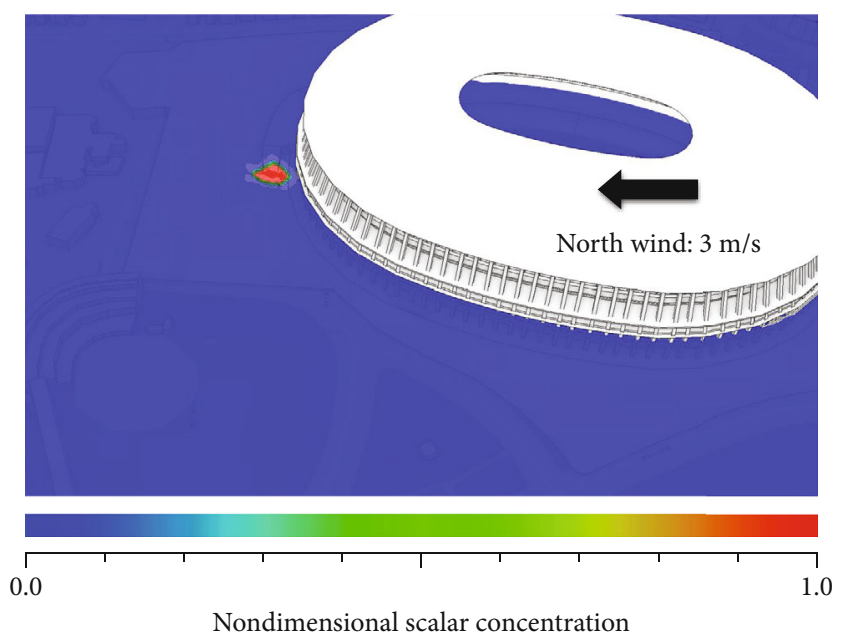

(a)

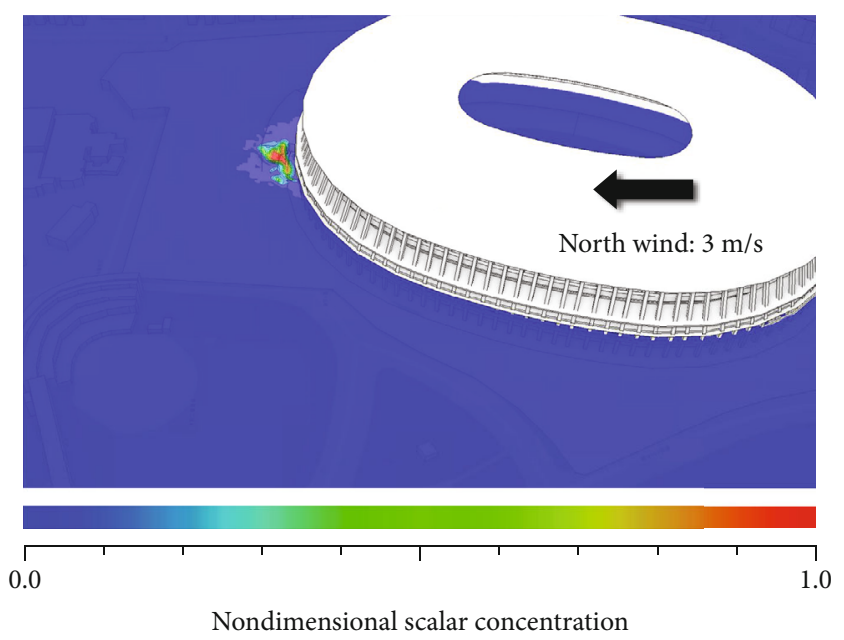

(b)

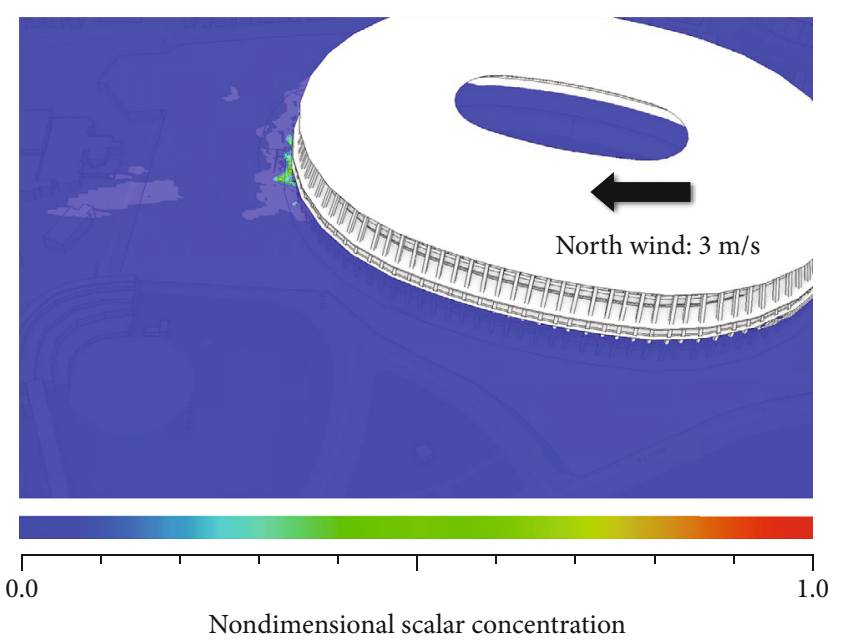

(c)

Figure 29: Time variation of the 2D scalar concentration field emitted from volume source A shown in Figure 27: (a) 20.00 seconds, (b) 120.00 seconds, and (c) 320.00 seconds after calculation of the scalar concentration field.

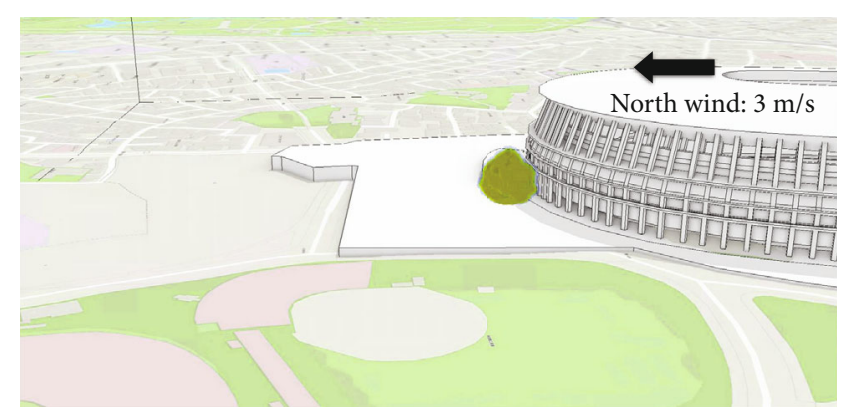

(a)

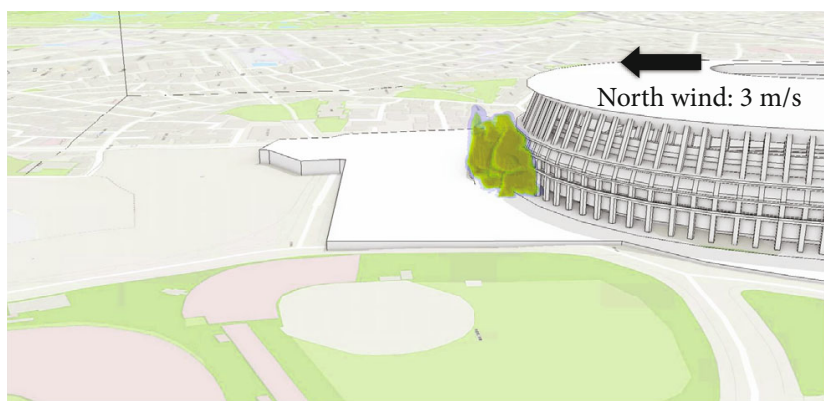

(b)

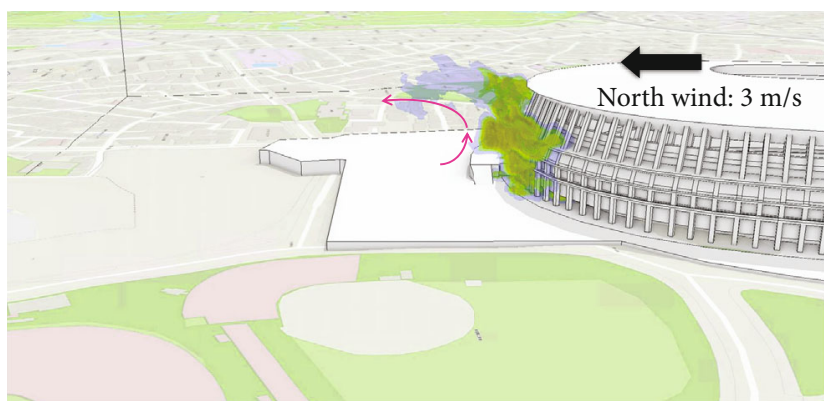

(c)

FIgURE 30: Time variation of the 3D scalar concentration field emitted from volume source A shown in Figure 27: (a) 20.00 seconds, (b) 120.00 seconds, and (c) 320.00 seconds after calculation of the scalar concentration field.

Finally, we tried to reproduce the hypothetical spread and dissipation of the fluid layer (assuming the fluid layer contains COVID-19 particles) outside the Japan National Stadium. Similar to the simulation shown above, we paid attention to the effect of ventilation driven by natural wind. As shown in Figure 26, we assumed that there were two volume sources (assuming the volume sources contain COVID19 particles) outside the Japan National Stadium. One involved setting the volume source within the near-wake region (see A in Figure 27), while the second volume source was set in the region of the flow around the side of the stadium (see B in Figure 27). First, we considered the flow field generated near the ground around the stadium. Figure 28 shows the spatial distribution of the instantaneous nondimensional $U$-velocity near the ground. We focused on the near-wake region behind the stadium, indicated by the blue line in Figure 28. Here, it can be seen that a relatively large recirculating region formed. On the other hand, in the region 


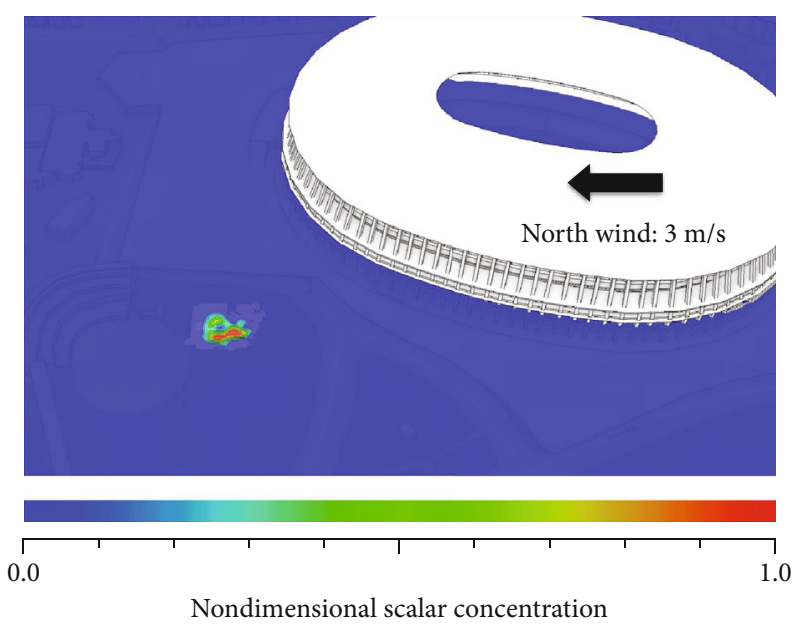

(a)

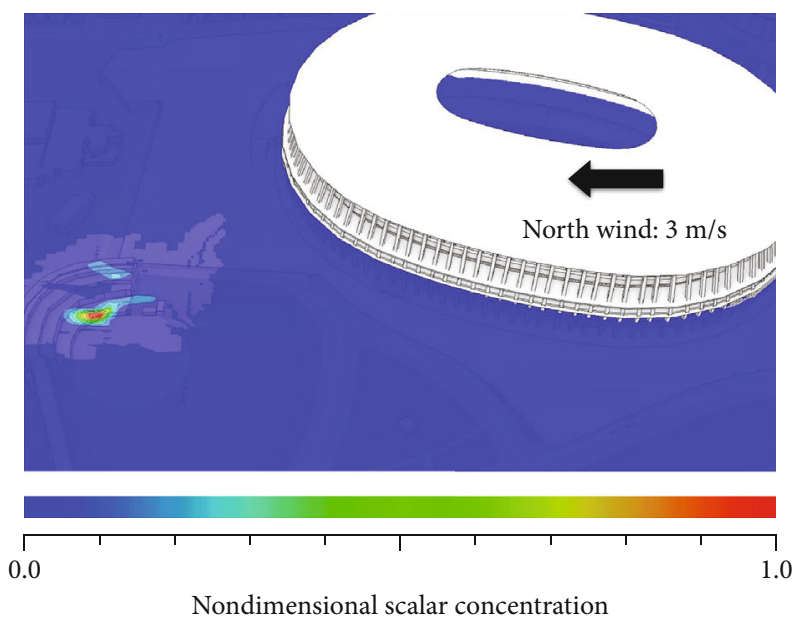

(b)

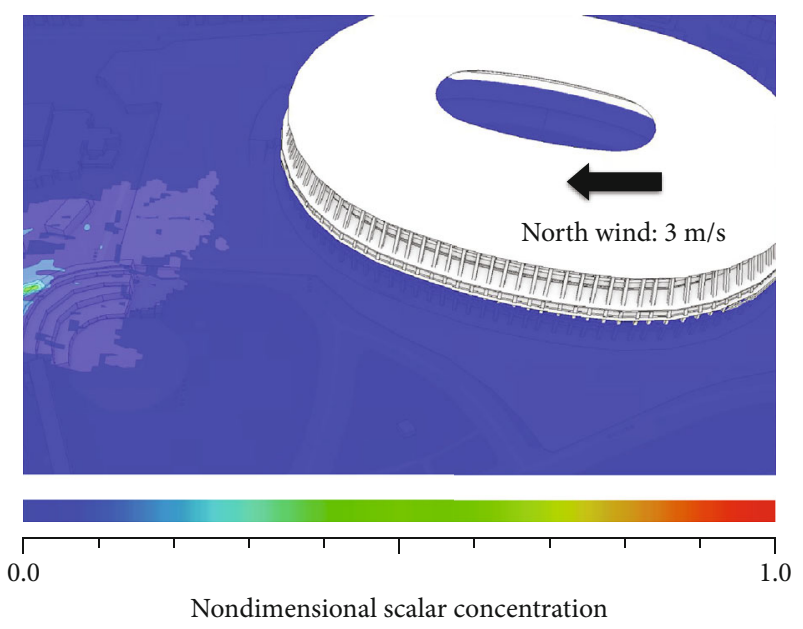

(c)

Figure 31: Time variation of the 2D scalar concentration field emitted from volume source B shown in Figure 27: (a) 20.00 seconds, (b) 120.00 seconds, and (c) 200.00 seconds after calculation of the scalar concentration field.

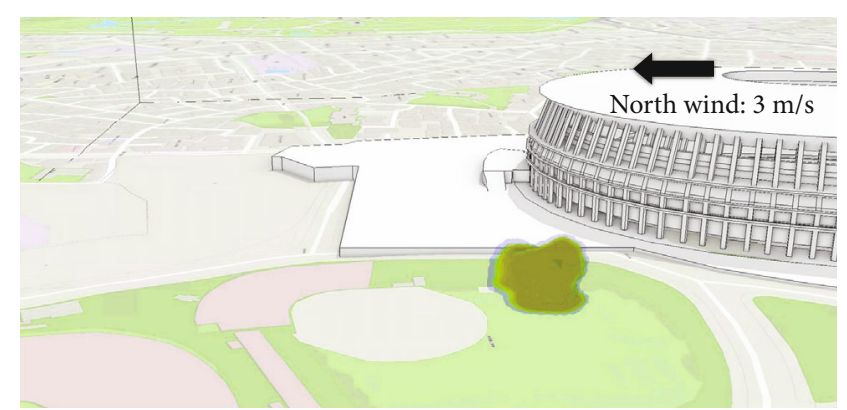

(a)

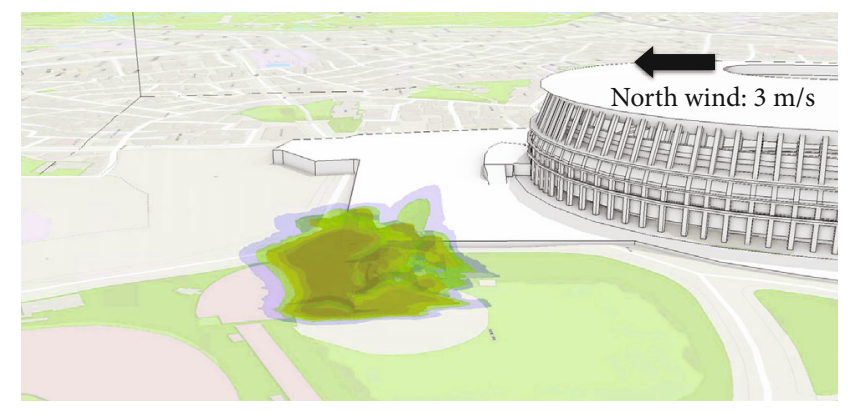

(b)

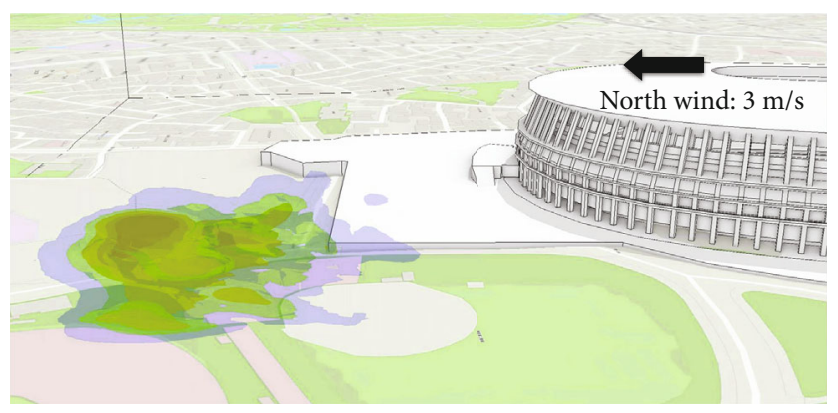

(c)

FIgURE 32: Time variation of the 3D scalar concentration field emitted from volume source B shown in Figure 27: (a) 20.00 seconds, (b) 120.00 seconds, and (c) 200.00 seconds after calculation of the scalar concentration field.

indicated by the red line in Figure 28, a locally accelerated flow around the stadium can be clearly observed.

Figure 29 displays the time variation of the 2D scalar concentration field emitted from volume source A shown in Figure 27. Figure 30 shows the time variation of the 3D scalar concentration field corresponding to Figure 29. Observing Figures 29 and 30 together shows the following: since the volume source was located within the region of flow around the side of the stadium, a scalar advection phenomenon occurred toward the stadium wall and then moved upward and was rapidly diffused downstream, as shown by the arrow in Figure 30(c). A series of passive scalar advection-diffusion phenomena occurred in a very short time.

Figure 31 presents the time variation of the $2 \mathrm{D}$ scalar concentration field emitted from volume source B shown in Figure 27. Figure 32 displays the time variation of the $3 \mathrm{D}$ scalar concentration field corresponding to Figure 31. Observing Figures 31 and 32 together shows the following: since the 
volume source was located within the region of flow around the side of the stadium, once released, the passive scalar concentration was rapidly diffused to the downstream side of the stadium.

\section{Conclusions}

In this paper, we applied the RIAM-COMPACT analysis function for gas diffusion, which was developed for complex terrain, using Airflow Analyst software and then used it to study the spread and dissipation of the fluid layer (assuming the fluid layer contains COVID-19 particles). First, to verify the prediction accuracy of the gas diffusion of RIAM-COMPACT, comparisons with past wind tunnel test results conducted on simple and complex terrains were presented under neutral atmospheric stability. The results of the numerical simulation carried out in this study showed good agreement with the wind tunnel experiment for both simple and complex terrain. Next, a model of the Japan National Stadium (Tokyo Olympic Stadium) was constructed using detailed 3D topographic AW3D data generated by combining high-resolution satellite images. We tried to reproduce the hypothetical spread and dissipation of the fluid layer (assuming the fluid layer contains COVID-19 particles) inside and outside the Japan National Stadium using Airflow Analyst implemented with the RIAM-COMPACT analysis function for gas diffusion. We paid special attention to the effect of ventilation driven by natural wind. For inside the stadium, as the initial condition, we set a situation where the volume source (assuming the volume source contains COVID-19 particles) exists on the entire ground. It should be noted that the accumulated value rapidly decreased about 300 seconds after the release of the scalar concentration due to the separated flow from the roof of the stadium. For outside the stadium, we assumed two volume sources (assuming the volume sources contain COVID-19 particles). One involved setting the volume source inside the near-wake region. The second volume source was set in the region of the flow around the side of the stadium. In both cases, it was clearly shown that once released, the passive scalar was rapidly diffused to the downstream side of the stadium. The numerical results, assuming various scenarios, ultimately demonstrated that ventilation driven by natural wind is very effective for the Japan National Stadium.

\section{Data Availability}

The data used to support the findings of this study are available from the corresponding author upon request.

\section{Conflicts of Interest}

All authors declare no conflicts of interest.

\section{Acknowledgments}

For conducting this research, the authors were provided with various types of data by NTT DATA Corporation and Remote Sensing Technology Center of Japan (RESTEC).
The authors would like to express their gratitude to all the organizations.

\section{References}

[1] W. Rodi, "Comparisons of LES and RANS calculations of the flow around bluff bodies," Journal of Wind Engineering and Industrial Aerodynamics, vol. 69-71, pp. 55-75, 1997.

[2] T. Stathopoulos, "Computational wind engineering: past achievements and future challenges," Journal of Wind Engineering and Industrial Aerodynamics, vol. 67-68, pp. 509532, 1997.

[3] R. N. Meroney, Wind tunnel and numerical simulation of pollution dispersion: a hybrid approach, in Lecture Series Proceeding from the Croucher Advanced Study Institute, Hong University of Science and Technology, Fort Collins, CO, 2004.

[4] J. Franke, C. Hirsch, G. Jensen et al., "Recommendations on the use of CFD in wind engineering," in Proceedings of the International Conference on Urban Wind Engineering and Building Aerodynamics, pp. C.1.1-C1.11, Belgium, 2004.

[5] J. Sumner, C. S. Watters, and C. Masson, "CFD in wind energy: the virtual, multiscale wind tunnel," Energies, vol. 3, no. 5, pp. 989-1013, 2010.

[6] L. Cochran and R. Derickson, "A physical modeler's view of Computational Wind Engineering," Journal of Wind Engineering and Industrial Aerodynamics, vol. 99, no. 4, pp. 139-153, 2011.

[7] B. Blocken, "50 years of computational wind engineering: past, present, and future," Journal of Wind Engineering and Industrial Aerodynamics, vol. 129, pp. 69-102, 2014.

[8] Y. Toparlar, B. Blocken, B. Maiheu, and G. J. F. van Heijst, “A review on the CFD analysis of urban microclimate," Renewable and Sustainable Energy Reviews, vol. 80, pp. 1613-1640, 2017.

[9] Á. Leelössy, I. Lagzi, A. Kovács, and R. Mészáros, “A review of numerical models to predict the atmospheric dispersion of radionuclides," Journal of Environmental Radioactivity, vol. 182, pp. 20-33, 2018.

[10] T. Uchida and K. Araki, "Reproduction of Local Strong Wind Area Induced in the Downstream of Small- Scale Terrain by Computational Fluid Dynamic (CFD) Approach," Modelling and Simulation in Engineering, vol. 2019, Article ID 7378315, 12 pages, 2019.

[11] T. Uchida and K. Sugitani, "Numerical and experimental study of topographic speed-up effects in complex terrain," Energies, vol. 13 , no. 15 , p. 3896, 2020.

[12] T. Uchida and Y. Kawashima, "New assessment scales for evaluating the degree of risk of wind turbine blade damage caused by terrain-induced turbulence," Energies, vol. 12, no. 13, p. 2624, 2019.

[13] T. Uchida, "Effects of inflow shear on wake characteristics of wind-turbines over flat terrain," Energies, vol. 13, no. 14, p. $3745,2020$.

[14] T. Uchida and Y. Ohya, "Numerical simulation of gas diffusion by using the RIAM-COMPACT. Part 1 . The case of an unstable condition," Reports of Research Institute for Applied Mechanics, Kyushu University, vol. 126, pp. 9-15, 2004.

[15] T. Uchida and Y. Ohya, "Numerical simulation of gas diffusion by using the RIAM-COMPACT. Part 2. The case of a stable condition," Reports of Research Institute for Applied Mechanics, Kyushu University, vol. 126, pp. 17-22, 2004. 
[16] https://www.esri.com/en-us/arcgis-marketplace/listing/ products/06effa0bee204361a28bc00d5258dae3.

[17] //http://www.esri.com/.

[18] https://www.aw3d.jp/.

[19] V. Vuorinen, M. Aarnio, M. Alava et al., "Modelling aerosol transport and virus exposure with numerical simulations in relation to SARS-CoV-2 transmission by inhalation indoors," Safety Science, vol. 130, article 104866, 2020.

[20] J. Kim and P. Moin, "Application of a fractional-step method to incompressible Navier-Stokes equations," Journal of Computational Physics, vol. 59, no. 2, pp. 308-323, 1985.

[21] T. Kajishima, "Upstream-shifted interpolation method for numerical simulation of incompressible flows," Transactions Of the Japan Society of Mechanical Engineers Series B, vol. 60, no. 578, pp. 3319-3326, 1994.

[22] T. Kawamura, H. Takami, and K. Kuwahara, "Computation of high Reynolds number flow around a circular cylinder with surface roughness," Fluid Dynamics Research, vol. 1, no. 2, pp. 145-162, 1986.

[23] J. Smagorinsky, "General circulation experiments with the primitive equations. I. Basic experiments," Monthly Weather Review, vol. 91, no. 3, pp. 99-164, 1963.

[24] W. H. Snyder and J. C. R. Hunt, "Turbulent diffusion from a point source in stratified and neutral flows around a threedimensional hill-II. Laboratory measurements of surface concentrations," Atmospheric Environment, vol. 18, no. 10, 1969.

[25] T. Hayashi, M. Chino, H. Yamazawa et al., "Effective stack heights obtained from wind tunnel and atmospheric diffusion experiments," Japan Atomic Energy Research Institute, 2001, JAERI-Tech 2001-034.

[26] https://www.jpnsport.go.jp/newstadium/. 